\title{
Five new pseudopolymorphs of sym-trinitrobenzene
}

Ram K. R. Jetti, ${ }^{\dagger}$ Roland Boese, ${ }^{*, \dagger}$ Praveen K. Thallapally, ${ }^{\ddagger}$ and Gautam R. Desiraju* ${ }^{*}$

Institut für Anorganische Chemie, Universität Duisburg-Essen, Standort

Essen,Universitätsstrasse 5-7, D-45177 Essen, Germany, School of Chemistry, University of Hyderabad, Hyderabad 500 046, India

\section{Supplementary Information}

(44 pages)

Ortep diagrams

Atomic coordinates

Bond lengths and angles

Anisotropic displacement parameters

DSC Thermograms

For structures 1-5 


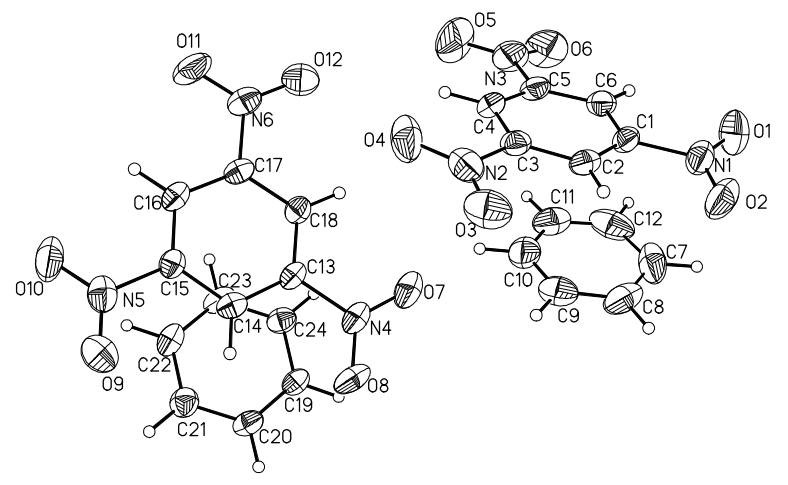

1

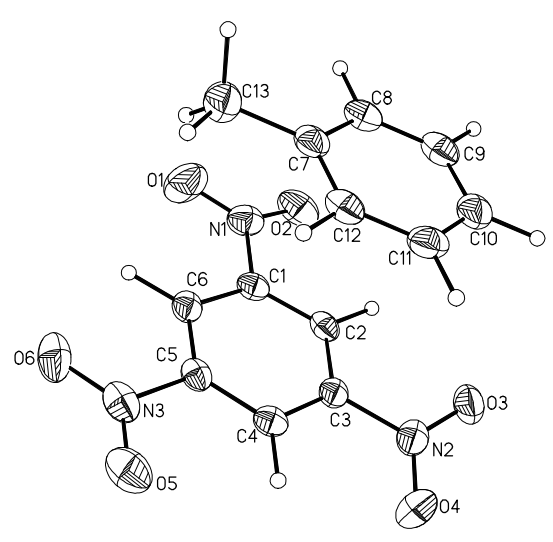

2

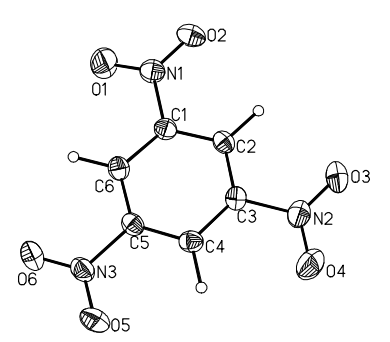

4

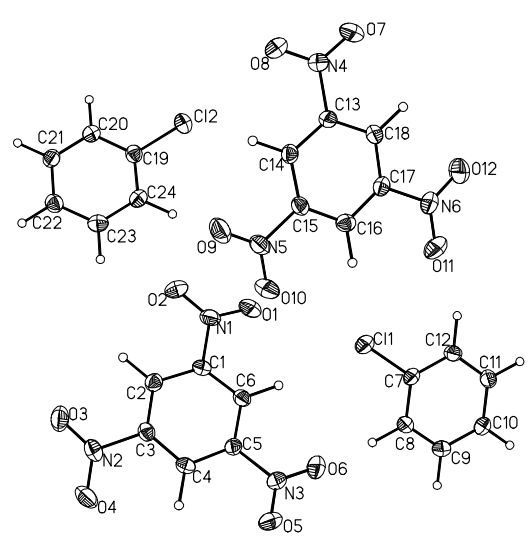

3

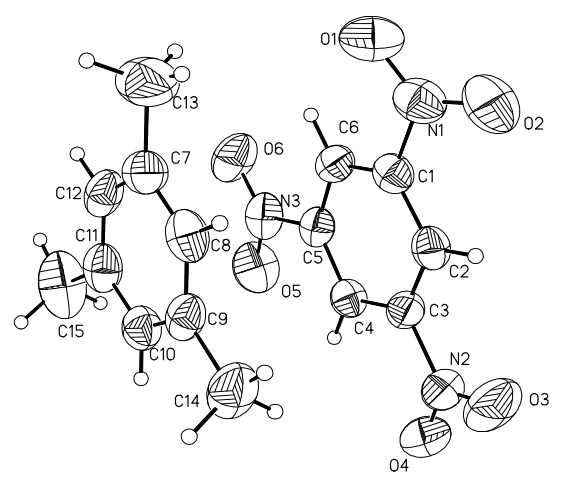


Table 1. Crystal data and structure refinement for 1 .

Identification code

Empirical formula

Formula weight

Density (calculated)

$\mathrm{F}(000)$

Temperature

Crystal size

Crystal color

Crystal description

Wavelength

Crystal system

Space group

Unit cell dimensions

Volume

$\mathrm{Z}$

Cell measurement reflections used

Cell measurement theta $\min / \max$

Diffractometer control software

Diffractometer measurement device

Diffractometer measurement method

Theta range for data collection

Completeness to theta $=28.60^{\circ}$

Index ranges

Computing data reduction

Absorption coefficient

Computing absorption correction

Absorption correction details

Max. / min. transmission

$\mathrm{R}$ (merg) before/after correction

Computing structure solution
1

$\mathrm{C}_{6} \mathrm{H}_{3} \mathrm{~N}_{3} \mathrm{O}_{6} * \mathrm{C}_{6} \mathrm{H}_{6}$

291.22 Da

$1.465 \mathrm{~g} \mathrm{~cm}^{-3}$

600

183(2) K

$0.57 \times 0.43 \times 0.18 \mathrm{~mm}$

yellow

plate

$0.71073 \AA$

triclinic

$P \overline{1}$

$a=7.189(5) \AA$

$\alpha=90.419(14)^{\circ}$

$b=12.620(8) \AA$

$\beta=103.320(14)^{\circ}$

$c=15.065(10) \AA$

$\gamma=96.598(13)^{\circ}$

4

3496

$2.78^{\circ}$ to $27.73^{\circ}$

Bruker AXS SMART Vers. 5.054 1997/98

Siemens SMART CCD area detector system

Full sphere data collection

in omega at $0.3^{\circ}$ scan width

two runs with 720 frames, phi $=0^{\circ}, 270^{\circ}$

and two runs with 436 frames, phi $=88^{\circ}, 180^{\circ}$

$2.18^{\circ}$ to $28.60^{\circ}$

$93.9 \%$

$-9<=\mathrm{h}<=9,-16<=\mathrm{k}<=16,-20<=1<=20$

Bruker AXS SAINT program Vers. 6.02A

$0.120 \mathrm{~mm}^{-1}$

Bruker AXS SADABS program multiscan V2.03

R.H. Blessing, Acta Cryst. (1995) A51 33-38

$0.979 / 0.925$

$0.209 / 0.100$

Bruker AXS SHELXTL Vers. 6.12 DOS/WIN95/NT/2000/ME 
Computing structure refinement

Refinement method

Reflections collected

Independent reflections

Data / restraints / parameters

Goodness-of-fit on $\mathrm{F}^{2}$

Weighting details

Final $\mathrm{R}$ indices [I $>2 \operatorname{sigma}(\mathrm{I})]$

$\mathrm{R}$ indices (all data)

Largest diff. peak and hole

Treatment of hydrogen atoms
Bruker AXS SHELXTL Vers. 6.12 DOS/WIN95/NT/2000/ME

Full-matrix least-squares on $\mathrm{F}^{2}$

15729

$6363[R($ int $)=0.0603]$

2795 / 0 / 379

0.870

$\mathrm{w}=1 /\left[\sigma^{2}\left(\mathrm{Fo}^{2}\right)+(\mathrm{a} 0.1088 * \mathrm{P})^{2}\right]$

where $\mathrm{P}=\left(\mathrm{Fo}^{2}+2 \mathrm{Fc}^{2}\right) / 3$

$R_{1}=0.0661, w R_{2}=0.1609$

$R_{1}=0.1420, w R_{2}=0.2016$

0.292 and $-0.233 \mathrm{e}^{-3}$

Riding model on idealized geometries

with the 1.2 fold isotropic displacement

parameters of the equivalent Uij of the

corresponding carbon atom 
Table 2. Atomic coordinates ( $\left.\times 10^{4}\right)$ and equivalent isotropic displacement parameters $\left(\AA^{2} \times 10^{3}\right)$ for $\mathbf{1}$. $U(e q)$ is defined as one third of the trace of the orthogonalized $U_{i j}$ tensor.

\begin{tabular}{|c|c|c|c|c|}
\hline & $\mathrm{x}$ & $\mathrm{y}$ & $\mathrm{z}$ & $\mathrm{U}(\mathrm{eq})$ \\
\hline $\mathrm{N}(1)$ & $3904(3)$ & $14676(2)$ & $1501(2)$ & $63(1)$ \\
\hline $\mathrm{N}(2)$ & $1872(3)$ & $11285(2)$ & $2745(2)$ & $64(1)$ \\
\hline $\mathrm{N}(3)$ & $3030(4)$ & $11367(2)$ & $-349(2)$ & $78(1)$ \\
\hline$O(1)$ & $4119(4)$ & $15163(2)$ & $831(2)$ & $94(1)$ \\
\hline$O(2)$ & $4087(3)$ & $15076(1)$ & $2264(2)$ & $86(1)$ \\
\hline$O(3)$ & $2122(3)$ & $11754(2)$ & $3486(2)$ & $88(1)$ \\
\hline$O(4)$ & $1181(3)$ & $10361(2)$ & $2569(2)$ & $89(1)$ \\
\hline$O(5)$ & $2476(4)$ & $10416(2)$ & $-426(2)$ & $123(1)$ \\
\hline$O(6)$ & $3663(4)$ & $11880(2)$ & $-908(2)$ & $103(1)$ \\
\hline $\mathrm{C}(1)$ & $3388(3)$ & $13508(2)$ & $1394(2)$ & $45(1)$ \\
\hline $\mathrm{C}(2)$ & $2947(3)$ & $12965(2)$ & $2117(2)$ & $47(1)$ \\
\hline$C(3)$ & 2425 (3) & $11884(2)$ & $1991(2)$ & $48(1)$ \\
\hline$C(4)$ & $2386(3)$ & $11346(2)$ & $1181(2)$ & $51(1)$ \\
\hline$C(5)$ & $2908(3)$ & $11931(2)$ & $505(2)$ & $51(1)$ \\
\hline$C(6)$ & $3396(3)$ & $13014(2)$ & $576(2)$ & $50(1)$ \\
\hline$C(7)$ & $8556(4)$ & $14124(2)$ & $1638(4)$ & $104(2)$ \\
\hline$C(8)$ & $8327(4)$ & $13731(3)$ & $2494(3)$ & $85(1)$ \\
\hline$C(9)$ & $7877(4)$ & $12681(3)$ & $2568(2)$ & $75(1)$ \\
\hline$C(10)$ & $7666(4)$ & $12004(2)$ & $1867(3)$ & $74(1)$ \\
\hline $\mathrm{C}(11)$ & $7879(4)$ & $12328(3)$ & $1064(3)$ & $78(1)$ \\
\hline $\mathrm{C}(12)$ & $8314(4)$ & $13347(4)$ & $911(2)$ & $92(1)$ \\
\hline$N(4)$ & $5926(3)$ & $9767(2)$ & $4017(2)$ & $52(1)$ \\
\hline$N(5)$ & 3441 (3) & $6187(2)$ & $4721(2)$ & $61(1)$ \\
\hline $\mathrm{N}(6)$ & 1517 (3) & $7499(2)$ & $1608(2)$ & $58(1)$ \\
\hline$O(7)$ & $5921(3)$ & $10448(1)$ & $3442(2)$ & $78(1)$ \\
\hline$O(8)$ & $6832(2)$ & $9878(1)$ & $4810(1)$ & $65(1)$ \\
\hline$O(9)$ & $4113(3)$ & $6408(2)$ & $5535(2)$ & $82(1)$ \\
\hline$O(10)$ & $2666(3)$ & $5307(1)$ & $4414(2)$ & $89(1)$ \\
\hline$O(11)$ & $603(3)$ & $6627(2)$ & $1372(1)$ & $82(1)$ \\
\hline$O(12)$ & 1495 (3) & $8265(2)$ & $1109(1)$ & 81 (1) \\
\hline$C(13)$ & $4754(3)$ & $8738(2)$ & $3717(2)$ & $41(1)$ \\
\hline
\end{tabular}




\begin{tabular}{lllll}
$C(14)$ & $4673(3)$ & $7981(2)$ & $4359(2)$ & $43(1)$ \\
$C(15)$ & $3557(3)$ & $7022(2)$ & $4058(2)$ & $42(1)$ \\
$C(16)$ & $2554(3)$ & $6834(2)$ & $3162(2)$ & $44(1)$ \\
$C(17)$ & $2671(3)$ & $7640(2)$ & $2557(2)$ & $44(1)$ \\
$C(18)$ & $3791(3)$ & $8600(2)$ & $2813(2)$ & $42(1)$ \\
$C(19)$ & $9625(3)$ & $8539(2)$ & $3930(2)$ & $46(1)$ \\
$C(20)$ & $9795(3)$ & $7956(2)$ & $4706(2)$ & $51(1)$ \\
$C(21)$ & $8966(3)$ & $6913(2)$ & $4654(2)$ & $54(1)$ \\
$C(22)$ & $7951(3)$ & $6458(2)$ & $3812(2)$ & $55(1)$ \\
$C(23)$ & $7770(3)$ & $7044(2)$ & $3033(2)$ & $53(1)$ \\
$C(24)$ & $8598(3)$ & $8095(2)$ & $3085(2)$ & $49(1)$ \\
\hline
\end{tabular}


Table 3. Bond lengths $[\AA]$ and angles $\left[^{\circ}\right]$ for $\mathbf{1}$.

\begin{tabular}{|c|c|c|c|}
\hline $\mathrm{N}(1)-\mathrm{O}(1)$ & $1.215(3)$ & $C(16)-C(17)$ & $1.379(3)$ \\
\hline$N(1)-O(2)$ & $1.225(3)$ & $C(17)-C(18)$ & $1.376(3)$ \\
\hline$N(1)-C(1)$ & $1.477(3)$ & $C(19)-C(20)$ & $1.375(4)$ \\
\hline$N(2)-O(4)$ & $1.217(3)$ & $C(19)-C(24)$ & $1.393(3)$ \\
\hline$N(2)-O(3)$ & $1.226(3)$ & $C(20)-C(21)$ & $1.375(3)$ \\
\hline$N(2)-C(3)$ & $1.473(3)$ & $C(21)-C(22)$ & $1.392(3)$ \\
\hline$N(3)-O(6)$ & $1.204(3)$ & $C(22)-C(23)$ & $1.381(4)$ \\
\hline$N(3)-O(5)$ & $1.217(3)$ & $C(23)-C(24)$ & $1.384(3)$ \\
\hline$N(3)-C(5)$ & $1.491(3)$ & & \\
\hline$C(1)-C(2)$ & $1.370(4)$ & $\mathrm{O}(1)-\mathrm{N}(1)-\mathrm{O}(2)$ & $125.3(2)$ \\
\hline$C(1)-C(6)$ & $1.378(3)$ & $\mathrm{O}(1)-\mathrm{N}(1)-\mathrm{C}(1)$ & $117.8(2)$ \\
\hline$C(2)-C(3)$ & $1.373(3)$ & $\mathrm{O}(2)-\mathrm{N}(1)-\mathrm{C}(1)$ & $116.9(2)$ \\
\hline$C(3)-C(4)$ & $1.385(3)$ & $\mathrm{O}(4)-\mathrm{N}(2)-\mathrm{O}(3)$ & $125.5(3)$ \\
\hline$C(4)-C(5)$ & $1.359(4)$ & $\mathrm{O}(4)-\mathrm{N}(2)-\mathrm{C}(3)$ & $116.4(2)$ \\
\hline$C(5)-C(6)$ & $1.369(3)$ & $O(3)-N(2)-C(3)$ & $118.1(2)$ \\
\hline$C(7)-C(8)$ & $1.422(6)$ & $O(6)-\mathrm{N}(3)-\mathrm{O}(5)$ & $125.0(3)$ \\
\hline$C(7)-C(12)$ & $1.432(5)$ & $O(6)-\mathrm{N}(3)-\mathrm{C}(5)$ & $118.2(2)$ \\
\hline$C(8)-C(9)$ & $1.340(5)$ & $O(5)-N(3)-C(5)$ & $116.8(3)$ \\
\hline$C(9)-C(10)$ & $1.322(4)$ & $C(2)-C(1)-C(6)$ & $123.2(2)$ \\
\hline$C(10)-C(11)$ & $1.317(5)$ & $\mathrm{C}(2)-\mathrm{C}(1)-\mathrm{N}(1)$ & $118.4(2)$ \\
\hline$C(11)-C(12)$ & $1.322(5)$ & $\mathrm{C}(6)-\mathrm{C}(1)-\mathrm{N}(1)$ & $118.5(2)$ \\
\hline$N(4)-O(8)$ & $1.221(3)$ & $C(1)-C(2)-C(3)$ & $117.1(2)$ \\
\hline $\mathrm{N}(4)-\mathrm{O}(7)$ & $1.225(3)$ & $C(2)-C(3)-C(4)$ & $122.3(2)$ \\
\hline$N(4)-C(13)$ & $1.471(3)$ & $C(2)-C(3)-N(2)$ & $118.3(2)$ \\
\hline$N(5)-O(10)$ & $1.220(3)$ & $\mathrm{C}(4)-\mathrm{C}(3)-\mathrm{N}(2)$ & $119.4(2)$ \\
\hline $\mathrm{N}(5)-\mathrm{O}(9)$ & $1.228(3)$ & $C(5)-C(4)-C(3)$ & $117.3(2)$ \\
\hline$N(5)-C(15)$ & $1.465(3)$ & $C(4)-C(5)-C(6)$ & $123.5(2)$ \\
\hline$N(6)-O(11)$ & $1.221(3)$ & $C(4)-C(5)-N(3)$ & $118.8(2)$ \\
\hline$N(6)-O(12)$ & $1.228(3)$ & $C(6)-C(5)-N(3)$ & $117.7(2)$ \\
\hline $\mathrm{N}(6)-\mathrm{C}(17)$ & $1.476(3)$ & $C(5)-C(6)-C(1)$ & $116.6(2)$ \\
\hline$C(13)-C(14)$ & $1.371(3)$ & $C(8)-C(7)-C(12)$ & $116.6(3)$ \\
\hline$C(13)-C(18)$ & $1.377(3)$ & $C(9)-C(8)-C(7)$ & $119.0(3)$ \\
\hline$C(14)-C(15)$ & $1.384(3)$ & $C(10)-C(9)-C(8)$ & $121.4(3)$ \\
\hline$C(15)-C(16)$ & $1.380(3)$ & $C(11)-C(10)-C(9)$ & $121.8(3)$ \\
\hline
\end{tabular}




$\begin{array}{ll}\mathrm{C}(10)-\mathrm{C}(11)-\mathrm{C}(12) & 122.1(3) \\ \mathrm{C}(11)-\mathrm{C}(12)-\mathrm{C}(7) & 119.2(3) \\ \mathrm{O}(8)-\mathrm{N}(4)-\mathrm{O}(7) & 125.0(2) \\ \mathrm{O}(8)-\mathrm{N}(4)-\mathrm{C}(13) & 118.2(2) \\ \mathrm{O}(7)-\mathrm{N}(4)-\mathrm{C}(13) & 116.9(2) \\ \mathrm{O}(10)-\mathrm{N}(5)-\mathrm{O}(9) & 124.4(2) \\ \mathrm{O}(10)-\mathrm{N}(5)-\mathrm{C}(15) & 116.6(2) \\ \mathrm{O}(9)-\mathrm{N}(5)-\mathrm{C}(15) & 118.9(2) \\ \mathrm{O}(11)-\mathrm{N}(6)-\mathrm{O}(12) & 123.8(2) \\ \mathrm{O}(11)-\mathrm{N}(6)-\mathrm{C}(17) & 117.5(2) \\ \mathrm{O}(12)-\mathrm{N}(6)-\mathrm{C}(17) & 118.6(2) \\ \mathrm{C}(14)-\mathrm{C}(13)-\mathrm{C}(18) & 124.11(19) \\ \mathrm{C}(14)-\mathrm{C}(13)-\mathrm{N}(4) & 117.8(2) \\ \mathrm{C}(18)-\mathrm{C}(13)-\mathrm{N}(4) & 118.1(2) \\ \mathrm{C}(13)-\mathrm{C}(14)-\mathrm{C}(15) & 116.5(2) \\ \mathrm{C}(16)-\mathrm{C}(15)-\mathrm{C}(14) & 122.1(2) \\ \mathrm{C}(16)-\mathrm{C}(15)-\mathrm{N}(5) & 119.51(19) \\ \mathrm{C}(14)-\mathrm{C}(15)-\mathrm{N}(5) & 118.3(2) \\ \mathrm{C}(17)-\mathrm{C}(16)-\mathrm{C}(15) & 118.23(19) \\ \mathrm{C}(18)-\mathrm{C}(17)-\mathrm{C}(16) & 122.1(2) \\ \mathrm{C}(18)-\mathrm{C}(17)-\mathrm{N}(6) & 118.0(2) \\ \mathrm{C}(16)-\mathrm{C}(17)-\mathrm{N}(6) & 119.92(19) \\ \mathrm{C}(17)-\mathrm{C}(18)-\mathrm{C}(13) & 116.9(2) \\ \mathrm{C}(20)-\mathrm{C}(19)-\mathrm{C}(24) & 121.1(2) \\ \mathrm{C}(21)-\mathrm{C}(20)-\mathrm{C}(19) & 120.0(2) \\ \mathrm{C}(20)-\mathrm{C}(21)-\mathrm{C}(22) & 119.5(2) \\ \mathrm{C}(23)-\mathrm{C}(22)-\mathrm{C}(21) & 120.6(2) \\ \mathrm{C}(22)-\mathrm{C}(23)-\mathrm{C}(24) & 120.0(2) \\ \mathrm{C}(23)-\mathrm{C}(24)-\mathrm{C}(19) & \end{array}$


Symmetry transformations used to generate equivalent atoms: 
Table 4. Anisotropic displacement parameters $\left(\AA^{2} \times 10^{3}\right)$ for $\mathbf{1}$. The anisotropic displacement factor exponent takes the form: $-2 \pi^{2}\left[h^{2} a^{* 2} U_{11}+\ldots+2 h k a^{*} b^{*} U_{12}\right]$

\begin{tabular}{|c|c|c|c|c|c|c|}
\hline & $\mathrm{U}_{11}$ & $\mathrm{U}_{22}$ & $\mathrm{U}_{33}$ & $\mathrm{U}_{23}$ & $\mathrm{U}_{13}$ & $\mathrm{U}_{12}$ \\
\hline $\mathrm{N}(1)$ & $53(1)$ & $44(1)$ & $89(2)$ & $-6(1)$ & $15(1)$ & $-1(1)$ \\
\hline $\mathrm{N}(2)$ & $53(1)$ & $74(2)$ & $68(2)$ & $22(1)$ & $15(1)$ & $11(1)$ \\
\hline$N(3)$ & $97(2)$ & $81(2)$ & $48(2)$ & $-20(1)$ & $0(1)$ & $12(1)$ \\
\hline$O(1) 1$ & $116(2)$ & $53(1)$ & $115(2)$ & $19(1)$ & $35(2)$ & $-3(1)$ \\
\hline$O(2)$ & $86(2)$ & $58(1)$ & $108(2)$ & $-33(1)$ & $18(1)$ & $-3(1)$ \\
\hline$O(3)$ & $88(2)$ & $121(2)$ & $58(1)$ & $13(1)$ & $22(1)$ & $15(1)$ \\
\hline$O(4)$ & $85(2)$ & $62(1)$ & $127(2)$ & $32(1)$ & $42(1)$ & $4(1)$ \\
\hline$O(5) 1$ & $186(3)$ & $80(2)$ & $92(2)$ & $-47(1)$ & $18(2)$ & $4(2)$ \\
\hline$O(6) 1$ & $133(2)$ & $128(2)$ & $47(2)$ & $-14(1)$ & $23(1)$ & $11(2)$ \\
\hline$C(1)$ & $37(1)$ & $41(1)$ & $54(2)$ & $-6(1)$ & $5(1)$ & $-2(1)$ \\
\hline$C(2)$ & $39(1)$ & $53(1)$ & $46(1)$ & $-8(1)$ & $2(1)$ & $3(1)$ \\
\hline$C(3)$ & $35(1)$ & $55(1)$ & $48(2)$ & $3(1)$ & $2(1)$ & $0(1)$ \\
\hline$C(4)$ & $44(1)$ & $45(1)$ & $54(2)$ & $-6(1)$ & $-3(1)$ & $-4(1)$ \\
\hline$C(5)$ & $50(1)$ & $55(1)$ & $39(1)$ & $-10(1)$ & $-3(1)$ & $1(1)$ \\
\hline$C(6)$ & $45(1)$ & $56(1)$ & $44(1)$ & $4(1)$ & $3(1)$ & $1(1)$ \\
\hline$C(7)$ & $43(2)$ & $56(2)$ & $202(5)$ & $36(2)$ & $9(2)$ & $-2(1)$ \\
\hline$C(8)$ & $56(2)$ & $89(2)$ & $99(3)$ & $-43(2)$ & $-1(2)$ & $9(2)$ \\
\hline$C(9)$ & $54(2)$ & $103(2)$ & $65(2)$ & $8(2)$ & $8(1)$ & $13(2)$ \\
\hline$C(10)$ & $51(2)$ & $67(2)$ & $96(3)$ & $1(2)$ & $6(2)$ & $5(1)$ \\
\hline$C(11)$ & $56(2)$ & $99(2)$ & $74(2)$ & $-20(2)$ & $2(2)$ & $12(2)$ \\
\hline$C(12)$ & $50(2)$ & $154(4)$ & $76(3)$ & $40(2)$ & $18(2)$ & $23(2)$ \\
\hline $\mathrm{N}(4)$ & $47(1)$ & $41(1)$ & $64(2)$ & $-9(1)$ & $14(1)$ & $-8(1)$ \\
\hline $\mathrm{N}(5)$ & $64(1)$ & $52(1)$ & $70(2)$ & $11(1)$ & $22(1)$ & $4(1)$ \\
\hline $\mathrm{N}(6)$ & $55(1)$ & $66(1)$ & $50(1)$ & $-13(1)$ & $8(1)$ & $-3(1)$ \\
\hline$O(7)$ & $82(1)$ & $49(1)$ & $91(2)$ & $2(1)$ & $12(1)$ & $-22(1)$ \\
\hline$O(8)$ & $55(1)$ & $63(1)$ & $67(1)$ & $-27(1)$ & $0(1)$ & $-9(1)$ \\
\hline$O(9)$ & $98(2)$ & $84(1)$ & $60(2)$ & $18(1)$ & $13(1)$ & $3(1)$ \\
\hline$O(10)$ & 112 (2) & $48(1)$ & $104(2)$ & $10(1)$ & $28(1)$ & $-14(1)$ \\
\hline$O(11)$ & ) $80(1)$ & $82(1)$ & $67(1)$ & $-26(1)$ & $-2(1)$ & $-24(1)$ \\
\hline$O(12)$ & 100 (2) & $85(1)$ & $47(1)$ & $-3(1)$ & $-2(1)$ & $3(1)$ \\
\hline$C(13)$ & ) $36(1)$ & $36(1)$ & $48(1)$ & $-8(1)$ & $10(1)$ & $-4(1)$ \\
\hline
\end{tabular}




\begin{tabular}{lccccc} 
C (14) $37(1)$ & $48(1)$ & $41(1)$ & $-8(1)$ & $8(1)$ & $3(1)$ \\
C (15) $40(1)$ & $39(1)$ & $48(1)$ & $1(1)$ & $14(1)$ & $0(1)$ \\
C (16) $37(1)$ & $41(1)$ & $55(2)$ & $-9(1)$ & $13(1)$ & $-3(1)$ \\
C (17) $38(1)$ & $50(1)$ & $40(1)$ & $-11(1)$ & $8(1)$ & $-1(1)$ \\
C (18) $41(1)$ & $41(1)$ & $45(1)$ & $0(1)$ & $13(1)$ & $-2(1)$ \\
C (19) $37(1)$ & $48(1)$ & $53(2)$ & $-7(1)$ & $12(1)$ & $-5(1)$ \\
$C(20) 42(1)$ & $59(1)$ & $47(2)$ & $-8(1)$ & $8(1)$ & $-8(1)$ \\
$C(21) 49(1)$ & $55(1)$ & $59(2)$ & $3(1)$ & $16(1)$ & $0(1)$ \\
$C(22) 52(2)$ & $43(1)$ & $72(2)$ & $-9(1)$ & $23(1)$ & $-5(1)$ \\
$C(23) 41(1)$ & $61(1)$ & $53(2)$ & $-19(1)$ & $12(1)$ & $-6(1)$ \\
$C(24) 41(1)$ & $59(1)$ & $47(2)$ & $-4(1)$ & $12(1)$ & $0(1)$ \\
\hline
\end{tabular}


Table 5. Hydrogen coordinates ( x 10 $0^{4}$ ) and isotropic displacement parameters $\left(\AA^{2} \times 10^{3}\right.$ ) for $\mathbf{1}$.

\begin{tabular}{|c|c|c|c|c|}
\hline & $\mathrm{x}$ & $\mathrm{y}$ & $\mathrm{z}$ & $\mathrm{U}(\mathrm{eq})$ \\
\hline $\mathrm{H}(2)$ & 3001 & 13321 & 2682 & 57 \\
\hline $\mathrm{H}(4)$ & 2012 & 10598 & 1102 & 61 \\
\hline $\mathrm{H}(6)$ & 3724 & 13406 & 87 & 60 \\
\hline $\mathrm{H}(7)$ & 8854 & 14864 & 1553 & 125 \\
\hline $\mathrm{H}(8)$ & 8490 & 14207 & 3005 & 102 \\
\hline $\mathrm{H}(9)$ & 7706 & 12416 & 3136 & 89 \\
\hline $\mathrm{H}(10)$ & 7351 & 11264 & 1946 & 89 \\
\hline $\mathrm{H}(11)$ & 7716 & 11815 & 580 & 94 \\
\hline $\mathrm{H}(12)$ & 8465 & 13560 & 325 & 110 \\
\hline $\mathrm{H}(14)$ & 5347 & 8107 & 4978 & 51 \\
\hline $\mathrm{H}(16)$ & 1804 & 6168 & 2968 & 53 \\
\hline H (18) & 3896 & 9143 & 2388 & 51 \\
\hline H (19) & 10218 & 9255 & 3971 & 56 \\
\hline $\mathrm{H}(20)$ & 10485 & 8274 & 5279 & 61 \\
\hline $\mathrm{H}(21)$ & 9086 & 6506 & 5188 & 65 \\
\hline $\mathrm{H}(22)$ & 7376 & 5738 & 3773 & 66 \\
\hline $\mathrm{H}(23)$ & 7078 & 6726 & 2461 & 63 \\
\hline $\mathrm{H}(24)$ & 8468 & 8508 & 2554 & 59 \\
\hline
\end{tabular}


Table 1. Crystal data and structure refinement for $\mathbf{2}$.

Identification code

Empirical formula

Formula weight

Density (calculated)

$\mathrm{F}(000)$

Temperature

Crystal size

Crystal color

Crystal description

Wavelength

Crystal system

Space group

Unit cell dimensions

Volume

$\mathrm{Z}$

Cell measurement reflections used

Cell measurement theta $\min / \max$

Diffractometer control software

Diffractometer measurement device

Diffractometer measurement method

Theta range for data collection

Completeness to theta $=28.48^{\circ}$

Index ranges

Computing data reduction

Absorption coefficient

Computing absorption correction

Absorption correction details

Max. / min. transmission

$\mathrm{R}$ (merg) before/after correction

Computing structure solution toldju $1 \mathrm{~m}$

$\mathrm{C}_{6} \mathrm{H}_{3} \mathrm{~N}_{3} \mathrm{O}_{6} * \mathrm{C}_{7} \mathrm{H}_{8}$

$305.25 \mathrm{Da}$

$1.496 \mathrm{~g} \mathrm{~cm}^{-3}$

632

183(2) K

$0.59 \times 0.43 \times 0.34 \mathrm{~mm}$

colorless

$\operatorname{rod}$

$0.71073 \AA$

monoclinic

$P 21 / c$

$a=7.279(2) \AA$

$\alpha=90^{\circ}$

$b=7.122(2) \AA$

$\beta=96.800(5)^{\circ}$

$c=26.337(8) \AA$

$\gamma=90^{\circ}$

$1355.6(7) \AA^{3}$

4

4528

$2.336^{\circ}$ to $28.298^{\circ}$

Bruker AXS SMART Vers. 5.054 1997/98

Siemens SMART CCD area detector system

Full sphere data collection

in omega at $0.3^{\circ}$ scan width

two runs with 720 frames, phi $=0^{\circ}, 270^{\circ}$

and two runs with 436 frames, phi $=88^{\circ}, 180^{\circ}$

$2.96^{\circ}$ to $28.48^{\circ}$

$98.4 \%$

$-9<=\mathrm{h}<=9,-9<=\mathrm{k}<=9,-35<=1<=34$

Bruker AXS SAINT program Vers. 6.02A

$0.121 \mathrm{~mm}^{-1}$

Bruker AXS SADABS program multiscan V2.03

R.H. Blessing, Acta Cryst. (1995) A51 33-38

$0.970 / 0.900$

$0.183 / 0.099$

Bruker AXS SHELXTL Vers. 6.12 DOS/WIN95/NT/2000/ME 
Computing structure refinement

Refinement method

Reflections collected

Independent reflections

Data / restraints / parameters

Goodness-of-fit on $\mathrm{F}^{2}$

Weighting details

Final $\mathrm{R}$ indices [I $>2 \operatorname{sigma}(\mathrm{I})]$

$\mathrm{R}$ indices (all data)

Largest diff. peak and hole

Treatment of hydrogen atoms

Comment
Bruker AXS SHELXTL Vers. 6.12 DOS/WIN95/NT/2000/ME

Full-matrix least-squares on $\mathrm{F}^{2}$

14233

$3386[R$ (int) $=0.0658]$

2144 / 0 / 199

0.983

$\mathrm{w}=1 /\left[\sigma^{2}\left(\mathrm{Fo}^{2}\right)+(0.1356 * \mathrm{P})^{2}\right]$

where $\mathrm{P}=\left(\mathrm{Fo}^{2}+2 \mathrm{Fc}^{2}\right) / 3$

$R_{1}=0.0787, w R_{2}=0.1902$

$R_{1}=0.1097, w R_{2}=0.2116$

0.495 and $-0.333 \mathrm{e}^{-3}$

Riding model on idealized geometries

with the 1.2 fold isotropic displacement

parameters of the equivalent $\mathrm{Uij}$ of the

corresponding carbon atom

Crystal unstable and turns opague immediately

and mounted with perfluorpolyether-oil and

cooled to $183 \mathrm{~K}$ 
Table 2. Atomic coordinates ( $\left.\times 10^{4}\right)$ and equivalent isotropic displacement parameters $\left(\AA^{2} \times 10^{3}\right)$ for $\mathbf{2}$. $U(e q)$ is defined as one third of the trace of the orthogonalized $U_{i j}$ tensor.

\begin{tabular}{|c|c|c|c|c|}
\hline & $\mathrm{x}$ & $\mathrm{y}$ & $\mathrm{z}$ & $\mathrm{U}(\mathrm{eq})$ \\
\hline $\mathrm{N}(1)$ & $3746(3)$ & $3332(3)$ & $261(1)$ & $50(1)$ \\
\hline $\mathrm{N}(2)$ & $4993(3)$ & $5706(3)$ & $1984(1)$ & $45(1)$ \\
\hline $\mathrm{N}(3)$ & $7020(3)$ & $-521(3)$ & $1588(1)$ & $47(1)$ \\
\hline$O(1)$ & $3782(4)$ & $2013(3)$ & $-21(1)$ & $79(1)$ \\
\hline$O(2)$ & $3063(3)$ & $4821(3)$ & $141(1)$ & $65(1)$ \\
\hline$O(3)$ & $3920(3)$ & $6960(2)$ & $1866(1)$ & $60(1)$ \\
\hline$O(4)$ & $5944(3)$ & $5587(3)$ & $2391(1)$ & $63(1)$ \\
\hline$O(5)$ & $7664(3)$ & $-651(3)$ & $2030(1)$ & $64(1)$ \\
\hline$O(6)$ & $7036(3)$ & $-1760(2)$ & $1278(1)$ & $62(1)$ \\
\hline$C(1)$ & $4562(3)$ & $3076(3)$ & $790(1)$ & $36(1)$ \\
\hline$C(2)$ & $4406(3)$ & $4503(3)$ & $1121(1)$ & $36(1)$ \\
\hline$C(3)$ & $5165(3)$ & $4230(3)$ & $1615(1)$ & $35(1)$ \\
\hline$C(4)$ & $6045(3)$ & $2619(3)$ & $1777(1)$ & $36(1)$ \\
\hline$C(5)$ & $6127(3)$ & $1236(3)$ & $1425(1)$ & $35(1)$ \\
\hline$C(6)$ & $5414(3)$ & $1416(3)$ & $926(1)$ & $37(1)$ \\
\hline$C(7)$ & $606(3)$ & $1041(3)$ & $1010(1)$ & $43(1)$ \\
\hline$C(8)$ & $-212(3)$ & $2569(3)$ & $758(1)$ & $45(1)$ \\
\hline$C(9)$ & $-484(3)$ & $4190(3)$ & $1004(1)$ & $48(1)$ \\
\hline$C(10)$ & $30(3)$ & $4332(3)$ & $1517(1)$ & $52(1)$ \\
\hline$C(11)$ & $839(3)$ & $2828(4)$ & $1776(1)$ & $50(1)$ \\
\hline$C(12)$ & $1118(3)$ & $1203(3)$ & $1526(1)$ & $45(1)$ \\
\hline$C(13)$ & $900(4)$ & $-731(4)$ & $739(1)$ & $60(1)$ \\
\hline
\end{tabular}


Table 3. Bond lengths $[\AA]$ and angles $\left[{ }^{\circ}\right]$ for 2 .

\begin{tabular}{|c|c|c|c|}
\hline$N(1)-O(2)$ & $1.198(3)$ & $C(6)-C(1)-N(1)$ & $118.76(19)$ \\
\hline$N(1)-O(1)$ & $1.201(3)$ & $C(1)-C(2)-C(3)$ & $116.83(19)$ \\
\hline $\mathrm{N}(1)-\mathrm{C}(1)$ & $1.459(3)$ & $C(4)-C(3)-C(2)$ & $122.88(18)$ \\
\hline$N(2)-O(3)$ & $1.203(3)$ & $C(4)-C(3)-N(2)$ & $118.44(18)$ \\
\hline$N(2)-O(4)$ & $1.208(3)$ & $C(2)-C(3)-N(2)$ & $118.66(19)$ \\
\hline$N(2)-C(3)$ & $1.449(3)$ & $C(3)-C(4)-C(5)$ & $117.06(18)$ \\
\hline$N(3)-O(6)$ & $1.204(3)$ & $C(4)-C(5)-C(6)$ & $123.19(19)$ \\
\hline$N(3)-O(5)$ & $1.206(3)$ & $C(4)-C(5)-N(3)$ & $118.52(18)$ \\
\hline$N(3)-C(5)$ & $1.451(3)$ & $C(6)-C(5)-N(3)$ & $118.29(19)$ \\
\hline$C(1)-C(2)$ & $1.352(3)$ & $C(5)-C(6)-C(1)$ & $116.52(19)$ \\
\hline$C(1)-C(6)$ & $1.363(3)$ & $C(12)-C(7)-C(8)$ & $117.6(2)$ \\
\hline$C(2)-C(3)$ & $1.365(3)$ & $C(12)-C(7)-C(13)$ & $120.9(2)$ \\
\hline$C(3)-C(4)$ & $1.358(3)$ & $C(8)-C(7)-C(13)$ & $121.5(2)$ \\
\hline$C(4)-C(5)$ & $1.359(3)$ & $C(9)-C(8)-C(7)$ & $121.7(2)$ \\
\hline$C(5)-C(6)$ & $1.360(3)$ & $C(8)-C(9)-C(10)$ & $120.1(2)$ \\
\hline$C(7)-C(12)$ & $1.371(3)$ & $C(9)-C(10)-C(11)$ & $119.3(2)$ \\
\hline$C(7)-C(8)$ & $1.373(3)$ & $C(12)-C(11)-C(10)$ & $120.4(2)$ \\
\hline$C(7)-C(13)$ & $1.478(3)$ & $C(11)-C(12)-C(7)$ & $120.9(2)$ \\
\hline$C(8)-C(9)$ & $1.350(3)$ & & \\
\hline$C(9)-C(10)$ & $1.361(4)$ & & \\
\hline$C(10)-C(11)$ & $1.365(4)$ & & \\
\hline$C(11)-C(12)$ & $1.358(3)$ & & \\
\hline $\mathrm{O}(2)-\mathrm{N}(1)-\mathrm{O}(1)$ & $124.6(2)$ & & \\
\hline $\mathrm{O}(2)-\mathrm{N}(1)-\mathrm{C}(1)$ & $118.2(2)$ & & \\
\hline $\mathrm{O}(1)-\mathrm{N}(1)-\mathrm{C}(1)$ & $117.2(2)$ & & \\
\hline $\mathrm{O}(3)-\mathrm{N}(2)-\mathrm{O}(4)$ & $124.59(19)$ & & \\
\hline$O(3)-N(2)-C(3)$ & $118.06(19)$ & & \\
\hline$O(4)-N(2)-C(3)$ & $117.35(19)$ & & \\
\hline$O(6)-\mathrm{N}(3)-\mathrm{O}(5)$ & $124.4(2)$ & & \\
\hline$O(6)-N(3)-C(5)$ & $118.1(2)$ & & \\
\hline$O(5)-N(3)-C(5)$ & $117.48(19)$ & & \\
\hline$C(2)-C(1)-C(6)$ & $123.50(18)$ & & \\
\hline $\mathrm{C}(2)-\mathrm{C}(1)-\mathrm{N}(1)$ & $117.73(19)$ & & \\
\hline
\end{tabular}


Symmetry transformations used to generate equivalent atoms: 
Table 4. Anisotropic displacement parameters $\left(\AA^{2} \times 10^{3}\right)$ for $\mathbf{2}$. The anisotropic displacement factor exponent takes the form: $-2 \pi^{2}\left[h^{2} a^{* 2} U_{11}+\ldots+2 h \mathrm{k}^{*} b^{*} U_{12}\right]$

\begin{tabular}{|c|c|c|c|c|c|c|}
\hline & $\mathrm{U}_{11}$ & $\mathrm{U}_{22}$ & $\mathrm{U}_{33}$ & $\mathrm{U}_{23}$ & $\mathrm{U}_{13}$ & $\mathrm{U}_{12}$ \\
\hline $\mathrm{N}(1)$ & $44(1)$ & $63(1)$ & $41(1)$ & $3(1)$ & $2(1)$ & $-4(1)$ \\
\hline $\mathrm{N}(2)$ & $41(1)$ & $42(1)$ & $54(1)$ & $-8(1)$ & $15(1)$ & $-8(1)$ \\
\hline $\mathrm{N}(3)$ & $38(1)$ & $41(1)$ & $63(1)$ & $13(1)$ & $13(1)$ & $1(1)$ \\
\hline$O(1) 1$ & $101(2)$ & $85(2)$ & $47(1)$ & $-12(1)$ & $-6(1)$ & $-1(1)$ \\
\hline$O(2)$ & $53(1)$ & $80(1)$ & $59(1)$ & $24(1)$ & $-3(1)$ & $12(1)$ \\
\hline$O(3)$ & $47(1)$ & $48(1)$ & $86(1)$ & $-17(1)$ & $10(1)$ & $9(1)$ \\
\hline$O(4)$ & $79(1)$ & $63(1)$ & $47(1)$ & $-13(1)$ & $3(1)$ & $-6(1)$ \\
\hline$O(5)$ & $60(1)$ & $56(1)$ & $73(1)$ & $24(1)$ & $1(1)$ & $8(1)$ \\
\hline$O(6)$ & $67(1)$ & $40(1)$ & $83(1)$ & $0(1)$ & $21(1)$ & $10(1)$ \\
\hline$C(1)$ & $27(1)$ & $44(1)$ & $38(1)$ & $5(1)$ & $4(1)$ & $-4(1)$ \\
\hline$C(2)$ & $25(1)$ & $35(1)$ & $48(1)$ & $4(1)$ & $6(1)$ & $1(1)$ \\
\hline$C(3)$ & $27(1)$ & $37(1)$ & $42(1)$ & $-4(1)$ & $10(1)$ & $-5(1)$ \\
\hline$C(4)$ & $28(1)$ & $42(1)$ & $39(1)$ & $5(1)$ & $6(1)$ & $-6(1)$ \\
\hline$C(5)$ & $27(1)$ & $32(1)$ & $47(1)$ & $5(1)$ & $10(1)$ & $-2(1)$ \\
\hline$C(6)$ & $30(1)$ & $38(1)$ & $44(1)$ & $-2(1)$ & $11(1)$ & $-3(1)$ \\
\hline$C(7)$ & $28(1)$ & $43(1)$ & $58(1)$ & $1(1)$ & $6(1)$ & $-5(1)$ \\
\hline$C(8)$ & $31(1)$ & $54(1)$ & $50(1)$ & $7(1)$ & $3(1)$ & $-4(1)$ \\
\hline$C(9)$ & $32(1)$ & $46(1)$ & $67(2)$ & $11(1)$ & $4(1)$ & $-2(1)$ \\
\hline$C(10)$ & ) 34 (1) & $47(1)$ & $75(2)$ & $-9(1)$ & $10(1)$ & $-6(1)$ \\
\hline$C(11)$ & ) 33 (1) & $66(2)$ & $49(1)$ & $-1(1)$ & $2(1)$ & $-8(1)$ \\
\hline$C(12)$ & ) 30 (1) & $48(1)$ & $56(1)$ & $9(1)$ & $3(1)$ & $-1(1)$ \\
\hline$C(13)$ & $54(2)$ & $52(2)$ & $74(2)$ & $-9(1)$ & $11(1)$ & $-1(1)$ \\
\hline
\end{tabular}


Table 5. Hydrogen coordinates ( x 104) and isotropic displacement parameters $\left(\AA^{2} \times 10^{3}\right.$ ) for 2.

\begin{tabular}{lrrrl}
\hline & $\mathrm{x}$ & $\mathrm{y}$ & $\mathrm{z}$ & $\mathrm{U}(\mathrm{eq})$ \\
& & & & \\
& & & & \\
$\mathrm{H}(2)$ & 3798 & 5642 & 1015 & 43 \\
$\mathrm{H}(4)$ & 6579 & 2466 & 2121 & 43 \\
$\mathrm{H}(6)$ & 5505 & 437 & 686 & 44 \\
$\mathrm{H}(8)$ & -596 & 2484 & 401 & 54 \\
$\mathrm{H}(9)$ & -1034 & 5233 & 819 & 58 \\
$\mathrm{H}(10)$ & -172 & 5464 & 1692 & 62 \\
$\mathrm{H}(11)$ & 1211 & 2919 & 2133 & 60 \\
$\mathrm{H}(12)$ & 1674 & 165 & 1711 & 54 \\
$\mathrm{H}(13 \mathrm{~A})$ & 1493 & -1652 & 981 & 90 \\
$\mathrm{H}(13 \mathrm{~B})$ & 1696 & -494 & 470 & 90 \\
$\mathrm{H}(13 \mathrm{C})$ & -295 & -1222 & 584 & 90 \\
& & & & \\
\hline
\end{tabular}


Table 1. Crystal data and structure refinement for $\mathbf{3}$.

Identification code

Empirical formula

Formula weight

Density (calculated)

$\mathrm{F}(000)$

Temperature

Crystal size

Crystal color

Crystal description

Wavelength

Crystal system

Space group

Unit cell dimensions

Volume

$\mathrm{Z}$

Diffractometer control software

Diffractometer measurement device

Diffractometer measurement method

Theta range for data collection

Completeness to theta $=28.24^{\circ}$

Index ranges

Computing data reduction

Absorption coefficient

Computing absorption correction

Absorption correction details

Max. / min. transmission

$\mathrm{R}$ (merg) before/after correction

Computing structure solution

Computing structure refinement

Refinement method
3

$\mathrm{C}_{6} \mathrm{H}_{3} \mathrm{~N}_{3} \mathrm{O}_{6} * \mathrm{C}_{6} \mathrm{H}_{5} \mathrm{Cl}$

$162.83 \mathrm{Da}$

$1.591 \mathrm{~g} \mathrm{~cm}^{-3}$

664

293(2) K

$0.53 \times 0.45 \times 0.31 \mathrm{~mm}$

colorless

block

$0.71073 \AA$

triclinic

$P \overline{1}$

$a=7.280(2) \AA$

$\alpha=88.75(3)^{\circ}$

$b=13.190(3) \AA$

$\beta=82.97(3)^{\circ}$

$c=14.520(3) \AA$

$\gamma=79.26(3)^{\circ}$

$1359.5(5) \AA^{3}$

8

Bruker AXS SMART Vers. 5.054 1997/98

Siemens SMART CCD area detector system

Full sphere data collection

in omega at $0.3^{\circ}$ scan width

two runs with 720 frames, phi $=0^{\circ}, 270^{\circ}$

and two runs with 436 frames, phi $=88^{\circ}, 180^{\circ}$

$2.83^{\circ}$ to $28.24^{\circ}$

$90.4 \%$

$-9<=\mathrm{h}<=9,-17<=\mathrm{k}<=15,-12<=\mathrm{l}<=19$

Bruker AXS SAINT program Vers. 6.02A

$0.316 \mathrm{~mm}^{-1}$

Bruker AXS SADABS program multiscan V2.03

R.H. Blessing, Acta Cryst. (1995) A51 33-38

$0.907 / 0.906$

$0.112 / 0.038$

Bruker AXS SHELXTL Vers. 6.10 DOS/WIN95/NT/2000

Bruker AXS SHELXTL Vers. 6.10 DOS/WIN95/NT/2000

Full-matrix least-squares on $\mathrm{F}^{2}$ 
Reflections collected

Independent reflections

Data / restraints / parameters

Goodness-of-fit on $\mathrm{F}^{2}$

Weighting details

Final $\mathrm{R}$ indices [I $>2 \operatorname{sigma}(\mathrm{I})]$

$\mathrm{R}$ indices (all data)

Largest diff. peak and hole

Treatment of hydrogen atoms
8720

$6087[R$ (int) $=0.0361]$

4345 / 0 / 397

1.024

$\mathrm{w}=1 /\left[\sigma^{2}\left(\mathrm{Fo}^{2}\right)+(0.0797 * \mathrm{P})^{2}\right]$

where $\mathrm{P}=\left(\mathrm{Fo}^{2}+2 \mathrm{Fc}^{2}\right) / 3$

$R 1=0.0530, w R_{2}=0.1292$

$R 1=0.0768, w R_{2}=0.1474$

0.673 and $-0.267 \mathrm{e}^{-3}$

Riding model on idealized geometries

with the 1.2 fold isotropic displacement

parameters of the equivalent Uij of the corresponding carbon atom 
Table 2. Atomic coordinates ( $\left.\times 10^{4}\right)$ and equivalent isotropic displacement parameters $\left(\AA^{2} \times 10^{3}\right)$ for 3. $U(e q)$ is defined as one third of the trace of the orthogonalized $U_{i j}$ tensor.

\begin{tabular}{|c|c|c|c|c|}
\hline & $\mathrm{x}$ & $\mathrm{y}$ & $\mathrm{z}$ & $\mathrm{U}(\mathrm{eq})$ \\
\hline $\mathrm{Cl}(1)$ & $1319(1)$ & $6156(1)$ & $9433(1)$ & $47(1)$ \\
\hline $\mathrm{N}(1)$ & $2465(3)$ & $5575(1)$ & $6468(1)$ & $44(1)$ \\
\hline$N(2)$ & $4558(2)$ & $8235(2)$ & $4388(1)$ & $43(1)$ \\
\hline $\mathrm{N}(3)$ & $3166(3)$ & $8879(1)$ & $7735(1)$ & $42(1)$ \\
\hline$O(1)$ & $1671(3)$ & $5387(1)$ & 7225 ( 1) & $57(1)$ \\
\hline$O(2)$ & $2869(3)$ & $4974(1)$ & $5818(1)$ & $63(1)$ \\
\hline$O(3)$ & $4999(3)$ & $7586(1)$ & $3779(1)$ & $58(1)$ \\
\hline$O(4)$ & $4583(3)$ & $9156(1)$ & $4279(1)$ & $63(1)$ \\
\hline$O(5)$ & $3992(3)$ & $9608(1)$ & $7621(1)$ & $60(1)$ \\
\hline$O(6)$ & $2340(3)$ & $8660(1)$ & $8474(1)$ & $60(1)$ \\
\hline $\mathrm{C}(1)$ & $2943(3)$ & $6615(1)$ & $6324(1)$ & $33(1)$ \\
\hline$C(2)$ & $3542(3)$ & $6905(2)$ & $5440(1)$ & $36(1)$ \\
\hline$C(3)$ & $3937(3)$ & $7891(1)$ & $5333(1)$ & $33(1)$ \\
\hline$C(4)$ & $3811(3)$ & $8559(1)$ & $6063(1)$ & $34(1)$ \\
\hline$C(5)$ & $3239(3)$ & $8208(1)$ & $6934(1)$ & $32(1)$ \\
\hline$C(6)$ & $2763(3)$ & $7242(1)$ & $7086(1)$ & $34(1)$ \\
\hline$C(7)$ & $41(3)$ & $6873(1)$ & $10374(1)$ & $34(1)$ \\
\hline$C(8)$ & $-551(3)$ & $7927(2)$ & $10267(1)$ & $37(1)$ \\
\hline$C(9)$ & $-1554(3)$ & $8494(2)$ & $11013(2)$ & $40(1)$ \\
\hline$C(10)$ & $-1968(3)$ & $8019(2)$ & $11854(2)$ & $38(1)$ \\
\hline $\mathrm{C}(11)$ & $-1381(3)$ & $6960(2)$ & $11946(2)$ & $40(1)$ \\
\hline $\mathrm{C}(12)$ & $-366(3)$ & $6379(1)$ & $11204(1)$ & $37(1)$ \\
\hline $\mathrm{Cl}(2)$ & $424(1)$ & $1224(1)$ & $5836(1)$ & $52(1)$ \\
\hline$N(4)$ & $7640(3)$ & $474(1)$ & 8461 (1) & $47(1)$ \\
\hline$N(5)$ & $6541(3)$ & $4076(1)$ & $7426(1)$ & $44(1)$ \\
\hline$N(6)$ & 3993 (3) & $3186(2)$ & $10562(1)$ & $45(1)$ \\
\hline$O(7)$ & $7344(3)$ & $-152(1)$ & $9067(1)$ & $76(1)$ \\
\hline$O(8)$ & $8590(3)$ & $253(1)$ & $7717(1)$ & $63(1)$ \\
\hline$O(9)$ & $7654(3)$ & $3811(1)$ & $6736(1)$ & $61(1)$ \\
\hline$O(10)$ & $5622(3)$ & $4948(1)$ & $7559(1)$ & $64(1)$ \\
\hline$O(11)$ & $3382(3)$ & $4106(1)$ & $10692(1)$ & $61(1)$ \\
\hline
\end{tabular}




\begin{tabular}{lllll} 
O(12) & $3804(3)$ & $2513(1)$ & $11134(1)$ & $70(1)$ \\
C (13) & $6801(3)$ & $1568(1)$ & $8647(1)$ & $35(1)$ \\
$C(14)$ & $7088(3)$ & $2278(1)$ & $7958(1)$ & $34(1)$ \\
C (15) & $6270(3)$ & $3297(1)$ & $8148(1)$ & $34(1)$ \\
C (16) & $5241(3)$ & $3618(2)$ & $8987(1)$ & $37(1)$ \\
C (17) & $5033(3)$ & $2870(2)$ & $9651(1)$ & $35(1)$ \\
C (18) & $5794(3)$ & $1832(2)$ & $9502(1)$ & $37(1)$ \\
C (19) & $1048(3)$ & $1945(2)$ & $4863(1)$ & $37(1)$ \\
$C(20)$ & $1348(3)$ & $1487(2)$ & $4000(2)$ & $38(1)$ \\
$C(21)$ & $1825(3)$ & $2064(2)$ & $3227(2)$ & $41(1)$ \\
$C(22)$ & $2014(3)$ & $3083(2)$ & $3328(2)$ & $42(1)$ \\
$C(23)$ & $1717(3)$ & $3527(2)$ & $4197(2)$ & $43(1)$ \\
$C(24)$ & $1226(3)$ & $2965(2)$ & $4979(2)$ & $40(1)$ \\
\hline
\end{tabular}


Table 3. Bond lengths $[\AA]$ and angles $\left[^{\circ}\right]$ for 3 .

\begin{tabular}{|c|c|c|c|}
\hline $\mathrm{Cl}(1)-\mathrm{C}(7)$ & $1.745(2)$ & $C(14)-C(15)$ & $1.383(3)$ \\
\hline $\mathrm{N}(1)-\mathrm{O}(2)$ & $1.219(2)$ & $C(15)-C(16)$ & $1.380(3)$ \\
\hline $\mathrm{N}(1)-\mathrm{O}(1)$ & $1.221(2)$ & $C(16)-C(17)$ & $1.380(3)$ \\
\hline $\mathrm{N}(1)-\mathrm{C}(1)$ & $1.481(2)$ & $C(17)-C(18)$ & $1.388(3)$ \\
\hline$N(2)-O(3)$ & $1.216(2)$ & $C(19)-C(20)$ & $1.378(3)$ \\
\hline $\mathrm{N}(2)-\mathrm{O}(4)$ & $1.225(2)$ & $C(19)-C(24)$ & $1.391(3)$ \\
\hline$N(2)-C(3)$ & $1.480(2)$ & $C(20)-C(21)$ & $1.387(3)$ \\
\hline$N(3)-O(6)$ & $1.220(2)$ & $C(21)-C(22)$ & $1.389(3)$ \\
\hline$N(3)-O(5)$ & $1.224(2)$ & $C(22)-C(23)$ & $1.378(3)$ \\
\hline$N(3)-C(5)$ & $1.467(3)$ & $C(23)-C(24)$ & $1.390(3)$ \\
\hline$C(1)-C(2)$ & $1.374(3)$ & & \\
\hline$C(1)-C(6)$ & $1.375(3)$ & $\mathrm{O}(2)-\mathrm{N}(1)-\mathrm{O}(1)$ & $124.74(17)$ \\
\hline$C(2)-C(3)$ & $1.385(3)$ & $\mathrm{O}(2)-\mathrm{N}(1)-\mathrm{C}(1)$ & $117.58(18)$ \\
\hline$C(3)-C(4)$ & $1.378(3)$ & $\mathrm{O}(1)-\mathrm{N}(1)-\mathrm{C}(1)$ & $117.67(18)$ \\
\hline$C(4)-C(5)$ & $1.379(3)$ & $\mathrm{O}(3)-\mathrm{N}(2)-\mathrm{O}(4)$ & $124.89(19)$ \\
\hline$C(5)-C(6)$ & $1.388(3)$ & $O(3)-N(2)-C(3)$ & $117.77(17)$ \\
\hline$C(7)-C(12)$ & $1.385(3)$ & $\mathrm{O}(4)-\mathrm{N}(2)-\mathrm{C}(3)$ & $117.34(19)$ \\
\hline$C(7)-C(8)$ & $1.388(3)$ & $O(6)-N(3)-O(5)$ & $124.37(19)$ \\
\hline$C(8)-C(9)$ & $1.381(3)$ & $O(6)-N(3)-C(5)$ & $118.35(17)$ \\
\hline$C(9)-C(10)$ & $1.385(3)$ & $O(5)-N(3)-C(5)$ & $117.25(17)$ \\
\hline$C(10)-C(11)$ & $1.391(3)$ & $C(2)-C(1)-C(6)$ & $123.32(17)$ \\
\hline$C(11)-C(12)$ & $1.387(3)$ & $\mathrm{C}(2)-\mathrm{C}(1)-\mathrm{N}(1)$ & $118.64(18)$ \\
\hline $\mathrm{Cl}(2)-\mathrm{C}(19)$ & $1.747(2)$ & $C(6)-C(1)-N(1)$ & $118.04(17)$ \\
\hline $\mathrm{N}(4)-\mathrm{O}(8)$ & $1.220(2)$ & $C(1)-C(2)-C(3)$ & $116.81(19)$ \\
\hline $\mathrm{N}(4)-\mathrm{O}(7)$ & $1.221(2)$ & $C(4)-C(3)-C(2)$ & $123.22(18)$ \\
\hline$N(4)-C(13)$ & $1.475(3)$ & $C(4)-C(3)-N(2)$ & $118.46(16)$ \\
\hline$N(5)-O(9)$ & $1.221(3)$ & $C(2)-C(3)-N(2)$ & $118.31(18)$ \\
\hline$N(5)-O(10)$ & $1.225(3)$ & $C(3)-C(4)-C(5)$ & $116.79(16)$ \\
\hline$N(5)-C(15)$ & $1.474(2)$ & $C(4)-C(5)-C(6)$ & $122.92(19)$ \\
\hline$N(6)-O(12)$ & $1.216(2)$ & $C(4)-C(5)-N(3)$ & $118.49(16)$ \\
\hline$N(6)-O(11)$ & $1.223(3)$ & $C(6)-C(5)-N(3)$ & $118.57(17)$ \\
\hline $\mathrm{N}(6)-\mathrm{C}(17)$ & $1.469(3)$ & $C(1)-C(6)-C(5)$ & $116.89(18)$ \\
\hline$C(13)-C(18)$ & $1.379(3)$ & $C(12)-C(7)-C(8)$ & $121.68(19)$ \\
\hline$C(13)-C(14)$ & $1.380(3)$ & $C(12)-C(7)-C 1(1)$ & $119.41(15)$ \\
\hline
\end{tabular}




\begin{tabular}{|c|c|}
\hline$C(8)-C(7)-C 1(1)$ & $118.90(16)$ \\
\hline$C(9)-C(8)-C(7)$ & $118.81(18)$ \\
\hline$C(8)-C(9)-C(10)$ & $120.61(18)$ \\
\hline$C(9)-C(10)-C(11)$ & $119.8(2)$ \\
\hline$C(12)-C(11)-C(10)$ & $120.39(19)$ \\
\hline$C(7)-C(12)-C(11)$ & $118.68(18)$ \\
\hline $\mathrm{O}(8)-\mathrm{N}(4)-\mathrm{O}(7)$ & $124.16(19)$ \\
\hline $\mathrm{O}(8)-\mathrm{N}(4)-\mathrm{C}(13)$ & $118.14(17)$ \\
\hline$O(7)-N(4)-C(13)$ & $117.69(19)$ \\
\hline$O(9)-N(5)-O(10)$ & $124.44(18)$ \\
\hline$O(9)-N(5)-C(15)$ & $118.22(18)$ \\
\hline$O(10)-N(5)-C(15)$ & $117.3(2)$ \\
\hline $\mathrm{O}(12)-\mathrm{N}(6)-\mathrm{O}(11)$ & $124.7(2)$ \\
\hline$O(12)-\mathrm{N}(6)-\mathrm{C}(17)$ & $117.64(19)$ \\
\hline $\mathrm{O}(11)-\mathrm{N}(6)-\mathrm{C}(17)$ & $117.67(18)$ \\
\hline$C(18)-C(13)-C(14)$ & $123.29(18)$ \\
\hline $\mathrm{C}(18)-\mathrm{C}(13)-\mathrm{N}(4)$ & $118.59(17)$ \\
\hline $\mathrm{C}(14)-\mathrm{C}(13)-\mathrm{N}(4)$ & $118.11(18)$ \\
\hline$C(13)-C(14)-C(15)$ & $116.85(19)$ \\
\hline$C(16)-C(15)-C(14)$ & $123.05(17)$ \\
\hline$C(16)-C(15)-N(5)$ & $118.65(18)$ \\
\hline$C(14)-C(15)-N(5)$ & $118.29(18)$ \\
\hline$C(15)-C(16)-C(17)$ & $117.09(18)$ \\
\hline$C(16)-C(17)-C(18)$ & $122.88(19)$ \\
\hline $\mathrm{C}(16)-\mathrm{C}(17)-\mathrm{N}(6)$ & $118.67(18)$ \\
\hline $\mathrm{C}(18)-\mathrm{C}(17)-\mathrm{N}(6)$ & $118.44(18)$ \\
\hline$C(13)-C(18)-C(17)$ & $116.82(18)$ \\
\hline$C(20)-C(19)-C(24)$ & $121.78(19)$ \\
\hline$C(20)-C(19)-C 1(2)$ & $119.05(15)$ \\
\hline$C(24)-C(19)-C 1(2)$ & $119.17(17)$ \\
\hline$C(19)-C(20)-C(21)$ & $118.97(19)$ \\
\hline$C(20)-C(21)-C(22)$ & $120.2(2)$ \\
\hline$C(23)-C(22)-C(21)$ & $120.1(2)$ \\
\hline$C(22)-C(23)-C(24)$ & $120.60(19)$ \\
\hline$C(23)-C(24)-C(19)$ & $118.3(2)$ \\
\hline
\end{tabular}


Symmetry transformations used to generate equivalent atoms: 
Table 4. Anisotropic displacement parameters $\left(\AA^{2} \times 10^{3}\right)$ for 3 . The anisotropic displacement factor exponent takes the form: $-2 \pi^{2}\left[h^{2} a^{* 2} U_{11}+\ldots+2 h k a^{*} b^{*} U_{12}\right]$

\begin{tabular}{|c|c|c|c|c|c|c|}
\hline & $\mathrm{U}_{11}$ & $\mathrm{U}_{22}$ & $\mathrm{U}_{33}$ & $\mathrm{U}_{23}$ & $\mathrm{U}_{13}$ & $\mathrm{U}_{12}$ \\
\hline $\mathrm{Cl}(1)$ & $57(1)$ & $39(1)$ & $41(1)$ & $-9(1)$ & $3(1)$ & $-5(1)$ \\
\hline $\mathrm{N}(1)$ & $54(1)$ & $29(1)$ & $50(1)$ & $5(1)$ & $-11(1)$ & $-9(1)$ \\
\hline $\mathrm{N}(2)$ & $43(1)$ & $52(1)$ & $35(1)$ & $11(1)$ & $-9(1)$ & $-14(1)$ \\
\hline $\mathrm{N}(3)$ & $51(1)$ & $32(1)$ & $40(1)$ & $-4(1)$ & $-2(1)$ & $-1(1)$ \\
\hline$O(1)$ & $75(1)$ & $39(1)$ & $59(1)$ & $13(1)$ & $-1(1)$ & $-20(1)$ \\
\hline$O(2)$ & $96(1)$ & $35(1)$ & $62(1)$ & $-10(1)$ & $-9(1)$ & $-20(1)$ \\
\hline$O(3)$ & $72(1)$ & $70(1)$ & $32(1)$ & $-3(1)$ & $-2(1)$ & $-19(1)$ \\
\hline$O(4)$ & $88(1)$ & $54(1)$ & $51(1)$ & $21(1)$ & $-10(1)$ & $-29(1)$ \\
\hline$O(5)$ & $86(1)$ & $37(1)$ & $58(1)$ & $-11(1)$ & $1(1)$ & $-22(1)$ \\
\hline$O(6)$ & $81(1)$ & $60(1)$ & $37(1)$ & $-7(1)$ & $8(1)$ & $-18(1)$ \\
\hline$C(1)$ & $35(1)$ & $26(1)$ & $38(1)$ & $4(1)$ & $-6(1)$ & $-5(1)$ \\
\hline$C(2)$ & $37(1)$ & $35(1)$ & $35(1)$ & $-2(1)$ & $-7(1)$ & $-5(1)$ \\
\hline$C(3)$ & $32(1)$ & $35(1)$ & $31(1)$ & $6(1)$ & $-6(1)$ & $-4(1)$ \\
\hline$C(4)$ & $32(1)$ & $27(1)$ & $40(1)$ & $5(1)$ & $-7(1)$ & $-4(1)$ \\
\hline$C(5)$ & $33(1)$ & $29(1)$ & $32(1)$ & $-1(1)$ & $-3(1)$ & $0(1)$ \\
\hline$C(6)$ & $36(1)$ & $30(1)$ & $35(1)$ & $6(1)$ & $-2(1)$ & $-2(1)$ \\
\hline$C(7)$ & $35(1)$ & $33(1)$ & $32(1)$ & $-6(1)$ & $-3(1)$ & $-4(1)$ \\
\hline$C(8)$ & $44(1)$ & $34(1)$ & $33(1)$ & $3(1)$ & $-3(1)$ & $-7(1)$ \\
\hline$C(9)$ & $44(1)$ & $31(1)$ & $43(1)$ & $0(1)$ & $-5(1)$ & $-4(1)$ \\
\hline$C(10)$ & $39(1)$ & $39(1)$ & $35(1)$ & $-6(1)$ & $-2(1)$ & $-6(1)$ \\
\hline$C(11)$ & $45(1)$ & $41(1)$ & $34(1)$ & $4(1)$ & $-8(1)$ & $-12(1)$ \\
\hline$C(12)$ & $43(1)$ & $30(1)$ & $40(1)$ & $3(1)$ & $-10(1)$ & $-7(1)$ \\
\hline $\mathrm{Cl}(2)$ & $62(1)$ & $52(1)$ & $41(1)$ & $8(1)$ & $1(1)$ & $-13(1)$ \\
\hline$N(4)$ & $65(1)$ & $34(1)$ & $40(1)$ & $-2(1)$ & $-9(1)$ & $0(1)$ \\
\hline $\mathrm{N}(5)$ & $53(1)$ & $39(1)$ & $43(1)$ & $11(1)$ & $-15(1)$ & $-13(1)$ \\
\hline$N(6)$ & $45(1)$ & $54(1)$ & $34(1)$ & $-7(1)$ & $-2(1)$ & $-7(1)$ \\
\hline$O(7) 1$ & $121(2)$ & $37(1)$ & $59(1)$ & $13(1)$ & $-1(1)$ & $4(1)$ \\
\hline$O(8)$ & $88(1)$ & $42(1)$ & $50(1)$ & $-11(1)$ & $3(1)$ & $3(1)$ \\
\hline$O(9)$ & $71(1)$ & $63(1)$ & $46(1)$ & $18(1)$ & $2(1)$ & $-15(1)$ \\
\hline$O(10)$ & $88(1)$ & $39(1)$ & $64(1)$ & $15(1)$ & $-19(1)$ & $-4(1)$ \\
\hline$O(11)$ & $66(1)$ & $55(1)$ & $56(1)$ & $-21(1)$ & $5(1)$ & $0(1)$ \\
\hline
\end{tabular}




\begin{tabular}{llllll}
$O(12) 90(1)$ & $70(1)$ & $40(1)$ & $7(1)$ & $14(1)$ & $-8(1)$ \\
$C(13) 42(1)$ & $30(1)$ & $34(1)$ & $0(1)$ & $-10(1)$ & $-1(1)$ \\
$C(14) 36(1)$ & $38(1)$ & $29(1)$ & $0(1)$ & $-7(1)$ & $-5(1)$ \\
$C(15) 36(1)$ & $35(1)$ & $32(1)$ & $5(1)$ & $-9(1)$ & $-8(1)$ \\
$C(16) 36(1)$ & $33(1)$ & $42(1)$ & $0(1)$ & $-12(1)$ & $-3(1)$ \\
$C(17) 36(1)$ & $41(1)$ & $30(1)$ & $-5(1)$ & $-5(1)$ & $-6(1)$ \\
$C(18) 44(1)$ & $36(1)$ & $31(1)$ & $4(1)$ & $-8(1)$ & $-8(1)$ \\
$C(19) 34(1)$ & $41(1)$ & $35(1)$ & $2(1)$ & $-2(1)$ & $-6(1)$ \\
$C(20) 39(1)$ & $36(1)$ & $39(1)$ & $-3(1)$ & $-3(1)$ & $-6(1)$ \\
$C(21) 41(1)$ & $41(1)$ & $37(1)$ & $-4(1)$ & $-3(1)$ & $-4(1)$ \\
$C(22) 39(1)$ & $43(1)$ & $42(1)$ & $4(1)$ & $-2(1)$ & $-6(1)$ \\
$C(23) 41(1)$ & $36(1)$ & $52(1)$ & $0(1)$ & $-7(1)$ & $-9(1)$ \\
$C(24) 41(1)$ & $40(1)$ & $39(1)$ & $-6(1)$ & $-5(1)$ & $-6(1)$ \\
\hline
\end{tabular}


Table 5. Hydrogen coordinates ( x 10 $0^{4}$ ) and isotropic displacement parameters $\left(\AA^{2} \times 10^{3}\right.$ ) for 3 .

\begin{tabular}{|c|c|c|c|c|}
\hline & $\mathrm{x}$ & $\mathrm{y}$ & $\mathrm{z}$ & $\mathrm{U}(\mathrm{eq})$ \\
\hline $\mathrm{H}(2)$ & 3677 & 6460 & 4938 & 43 \\
\hline $\mathrm{H}(4)$ & 4097 & 9215 & 5974 & 40 \\
\hline $\mathrm{H}(6)$ & 2342 & 7029 & 7677 & 41 \\
\hline $\mathrm{H}(8)$ & -276 & 8246 & 9702 & 44 \\
\hline $\mathrm{H}(9)$ & -1955 & 9201 & 10952 & 48 \\
\hline $\mathrm{H}(10)$ & -2637 & 8407 & 12355 & 46 \\
\hline $\mathrm{H}(11)$ & -1670 & 6640 & 12508 & 47 \\
\hline $\mathrm{H}(12)$ & 32 & 5671 & 11264 & 44 \\
\hline $\mathrm{H}(14)$ & 7797 & 2082 & 7392 & 41 \\
\hline $\mathrm{H}(16)$ & 4711 & 4308 & 9100 & 44 \\
\hline $\mathrm{H}(18)$ & 5633 & 1339 & 9958 & 44 \\
\hline $\mathrm{H}(20)$ & 1232 & 803 & 3937 & 46 \\
\hline $\mathrm{H}(21)$ & 2018 & 1768 & 2639 & 49 \\
\hline $\mathrm{H}(22)$ & 2341 & 3466 & 2809 & 50 \\
\hline H (23) & 1847 & 4210 & 4260 & 52 \\
\hline $\mathrm{H}(24)$ & 1022 & 3263 & 5566 & 48 \\
\hline
\end{tabular}


Table 1. Crystal data and structure refinement for 4 .

Identification code

Empirical formula

Formula weight

Density (calculated)

$\mathrm{F}(000)$

Temperature

Crystal size

Crystal color

Crystal description

Wavelength

Crystal system

Space group

Unit cell dimensions

Volume

$\mathrm{Z}$

Cell measurement reflections used

Cell measurement theta $\min / \max$

Diffractometer control software

Diffractometer measurement device

Diffractometer measurement method

Theta range for data collection

Completeness to theta $=28.34^{\circ}$

Index ranges

Computing data reduction

Absorption coefficient

Computing absorption correction

Absorption correction details

Max. / min. transmission

$\mathrm{R}$ (merg) before/after correction

Computing structure solution
4

$\mathrm{C}_{6} \mathrm{H}_{3} \mathrm{~N}_{3} \mathrm{O}_{6} * \mathrm{C}_{8} \mathrm{H}_{10}$

$319.27 \mathrm{Da}$

$1.422 \mathrm{~g} \mathrm{~cm}^{-3}$

664

183(2) K

$0.53 \times 0.38 \times 0.21 \mathrm{~mm}$

yellow

block

$0.71073 \AA$

monoclinic

$P 2_{1} / c$

$a=7.219(2) \AA$

$\alpha=90^{\circ}$

$b=28.485(9) \AA$

$\beta=93.404(6)^{\circ}$

$c=7.262(2) \AA$

$\gamma=90^{\circ}$

4

5073

$2.145^{\circ}$ to $28.150^{\circ}$

Bruker AXS SMART Vers. 5.054 1997/98

Siemens SMART CCD area detector system

Fullsphere data collection

in omega at $0.3^{\circ}$ scan width

two runs with 720 frames, phi $=0^{\circ}, 270^{\circ}$

and two runs with 436 frames, phi $=88^{\circ}, 180^{\circ}$

$2.83^{\circ}$ to $28.34^{\circ}$

$98.1 \%$

$-9<=\mathrm{h}<=9,-38<=\mathrm{k}<=37,-9<=\mathrm{l}<=9$

Bruker AXS SAINT program Vers. 6.02A

$0.113 \mathrm{~mm}^{-1}$

Bruker AXS SADABS program multiscan V2.03

R.H. Blessing, Acta Cryst. (1995) A51 33-38

0.977 / 0.938

$0.172 / 0.0984$

Bruker AXS SHELXTL Vers. 6.10 DOS/WIN95/NT/2000 
Computing structure refinement

Refinement method

Reflections collected

Independent reflections

Data / restraints / parameters

Goodness-of-fit on $\mathrm{F}^{2}$

Weighting details

Final $\mathrm{R}$ indices [I $>2 \operatorname{sigma}(\mathrm{I})]$

$\mathrm{R}$ indices (all data)

Largest diff. peak and hole

Treatment of hydrogen atoms

Comment
Bruker AXS SHELXTL Vers. 6.10 DOS/WIN95/NT/2000

Full-matrix least-squares on $\mathrm{F}^{2}$

12338

$3651[R($ int $)=0.0676]$

2497 / 0 / 208

0.975

$\mathrm{w}=1 /\left[\sigma^{2}\left(\mathrm{Fo}^{2}\right)+(0.1408 * \mathrm{P})^{2}\right]$

where $\mathrm{P}=\left(\mathrm{Fo}^{2}+2 \mathrm{Fc}^{2}\right) / 3$

$R_{1}=0.0681, w R_{2}=0.1812$

$R_{1}=0.0894, w R_{2}=0.1993$

0.401 and $-0.405 \mathrm{e}^{-3}$

Riding model on idealized geometries

with the 1.2 fold (1.5 fold for methyl

groups) isotropic displacement

parameters of the equivalent $\mathrm{Uij}$ of the corresponding carbon atom

Crystal unstable and turns opague immediately, so mounted with Perfluorpolyether-oil and cooled to $183 \mathrm{~K}$ 
Table 2. Atomic coordinates ( $\left.\times 10^{4}\right)$ and equivalent isotropic displacement parameters $\left(\AA^{2} \times 10^{3}\right)$ for 4 . $U(e q)$ is defined as one third of the trace of the orthogonalized $U_{i j}$ tensor.

\begin{tabular}{|c|c|c|c|c|}
\hline & $\mathrm{x}$ & $\mathrm{y}$ & $\mathrm{z}$ & $\mathrm{U}(\mathrm{eq})$ \\
\hline$N(1)$ & $965(2)$ & $3110(1)$ & $5553(2)$ & $50(1)$ \\
\hline $\mathrm{N}(2)$ & $1383(2)$ & $4734(1)$ & $3386(2)$ & $52(1)$ \\
\hline$N(3)$ & $-1342(2)$ & $3524(1)$ & $-745(2)$ & $45(1)$ \\
\hline$O(1)$ & $304(3)$ & $2722(1)$ & $5301(2)$ & $86(1)$ \\
\hline$O(2)$ & $1899(2)$ & $3227(1)$ & $6930(2)$ & $60(1)$ \\
\hline$O(3)$ & $2021(2)$ & $4839(1)$ & $4923(2)$ & $66(1)$ \\
\hline$O(4)$ & $1221(3)$ & $5010(1)$ & $2093(2)$ & $80(1)$ \\
\hline$O(5)$ & $-1431(2)$ & $3824(1)$ & $-1959(2)$ & $64(1)$ \\
\hline$O(6)$ & $-1903(2)$ & $3123(1)$ & $-936(2)$ & $60(1)$ \\
\hline$C(1)$ & $615(2)$ & $3465(1)$ & $4084(2)$ & $37(1)$ \\
\hline$C(2)$ & $1141(2)$ & $3922(1)$ & $4448(2)$ & $37(1)$ \\
\hline$C(3)$ & $818(2)$ & 4246 (1) & $3048(2)$ & $38(1)$ \\
\hline$C(4)$ & $-6(2)$ & $4125(1)$ & $1336(2)$ & $39(1)$ \\
\hline$C(5)$ & $-492(2)$ & $3662(1)$ & $1073(2)$ & $36(1)$ \\
\hline$C(6)$ & $-211(2)$ & $3321(1)$ & $2411(2)$ & $38(1)$ \\
\hline$C(7)$ & $5182(2)$ & $5948(1)$ & $9069(2)$ & $46(1)$ \\
\hline$C(8)$ & $5422(2)$ & $6429(1)$ & $8917(2)$ & $46(1)$ \\
\hline$C(9)$ & $4964(2)$ & $6672(1)$ & $7287(2)$ & $48(1)$ \\
\hline$C(10)$ & $4271(2)$ & $6415(1)$ & $5766(2)$ & $47(1)$ \\
\hline$C(11)$ & $4014(2)$ & $5936(1)$ & $5892(2)$ & $48(1)$ \\
\hline$C(12)$ & $4457(2)$ & $5700(1)$ & $7518(2)$ & $49(1)$ \\
\hline$C(13)$ & $5675(3)$ & $5696(1)$ & $10861(3)$ & $67(1)$ \\
\hline$C(14)$ & $5185(3)$ & $7197(1)$ & $7180(3)$ & $71(1)$ \\
\hline
\end{tabular}


Table 3. Bond lengths $[\AA]$ and angles $\left[^{\circ}\right]$ for 4.

\begin{tabular}{|c|c|c|c|}
\hline $\mathrm{N}(1)-\mathrm{O}(1)$ & $1.213(2)$ & $C(2)-C(1)-N(1)$ & $118.29(13)$ \\
\hline $\mathrm{N}(1)-\mathrm{O}(2)$ & $1.2189(19)$ & $C(6)-C(1)-N(1)$ & $118.52(14)$ \\
\hline $\mathrm{N}(1)-\mathrm{C}(1)$ & $1.480(2)$ & $C(1)-C(2)-C(3)$ & $117.20(13)$ \\
\hline $\mathrm{N}(2)-\mathrm{O}(3)$ & $1.2192(19)$ & $C(2)-C(3)-C(4)$ & $122.59(15)$ \\
\hline $\mathrm{N}(2)-\mathrm{O}(4)$ & $1.2246(19)$ & $C(2)-C(3)-N(2)$ & $118.46(13)$ \\
\hline $\mathrm{N}(2)-\mathrm{C}(3)$ & $1.465(2)$ & $C(4)-C(3)-N(2)$ & $118.95(14)$ \\
\hline $\mathrm{N}(3)-\mathrm{O}(6)$ & $1.219(2)$ & $C(5)-C(4)-C(3)$ & $116.94(14)$ \\
\hline$N(3)-O(5)$ & $1.2260(19)$ & $C(4)-C(5)-C(6)$ & $123.49(13)$ \\
\hline$N(3)-C(5)$ & $1.4754(18)$ & $C(4)-C(5)-N(3)$ & $117.92(13)$ \\
\hline$C(1)-C(2)$ & $1.379(2)$ & $C(6)-C(5)-N(3)$ & $118.58(14)$ \\
\hline$C(1)-C(6)$ & $1.384(2)$ & $C(5)-C(6)-C(1)$ & $116.58(15)$ \\
\hline$C(2)-C(3)$ & $1.382(2)$ & $C(8)-C(7)-C(12)$ & $118.40(15)$ \\
\hline$C(3)-C(4)$ & $1.389(2)$ & $C(8)-C(7)-C(13)$ & $120.99(16)$ \\
\hline$C(4)-C(5)$ & $1.376(2)$ & $C(12)-C(7)-C(13)$ & $120.61(18)$ \\
\hline$C(5)-C(6)$ & $1.381(2)$ & $C(7)-C(8)-C(9)$ & $122.31(15)$ \\
\hline$C(7)-C(8)$ & $1.386(3)$ & $C(10)-C(9)-C(8)$ & $117.85(18)$ \\
\hline$C(7)-C(12)$ & $1.404(2)$ & $C(10)-C(9)-C(14)$ & $121.02(17)$ \\
\hline$C(7)-C(13)$ & $1.511(2)$ & $C(8)-C(9)-C(14)$ & $121.12(16)$ \\
\hline$C(8)-C(9)$ & $1.395(2)$ & $C(11)-C(10)-C(9)$ & $120.70(16)$ \\
\hline$C(9)-C(10)$ & $1.393(2)$ & $C(12)-C(11)-C(10)$ & $120.85(15)$ \\
\hline$C(9)-C(14)$ & $1.504(3)$ & $C(11)-C(12)-C(7)$ & $119.88(17)$ \\
\hline$C(10)-C(11)$ & $1.381(3)$ & & \\
\hline$C(11)-C(12)$ & $1.380(2)$ & & \\
\hline $\mathrm{O}(1)-\mathrm{N}(1)-\mathrm{O}(2)$ & $124.50(15)$ & & \\
\hline $\mathrm{O}(1)-\mathrm{N}(1)-\mathrm{C}(1)$ & $117.64(14)$ & & \\
\hline $\mathrm{O}(2)-\mathrm{N}(1)-\mathrm{C}(1)$ & $117.86(14)$ & & \\
\hline$O(3)-N(2)-O(4)$ & $123.93(16)$ & & \\
\hline$O(3)-N(2)-C(3)$ & $118.17(14)$ & & \\
\hline$O(4)-N(2)-C(3)$ & $117.88(14)$ & & \\
\hline$O(6)-N(3)-O(5)$ & $124.64(14)$ & & \\
\hline$O(6)-N(3)-C(5)$ & $118.00(14)$ & & \\
\hline$O(5)-N(3)-C(5)$ & $117.36(14)$ & & \\
\hline$C(2)-C(1)-C(6)$ & $123.18(14)$ & & \\
\hline
\end{tabular}


Symmetry transformations used to generate equivalent atoms: 
Table 4. Anisotropic displacement parameters $\left(\AA^{2} \times 10^{3}\right)$ for 4 . The anisotropic displacement factor exponent takes the form: $-2 \pi^{2}\left[h^{2} a^{* 2} U_{11}+\ldots+2 h \mathrm{k}^{*} b^{*} U_{12}\right]$

\begin{tabular}{|c|c|c|c|c|c|c|}
\hline & $\mathrm{U}_{11}$ & $\mathrm{U}_{22}$ & $\mathrm{U}_{33}$ & $\mathrm{U}_{23}$ & $\mathrm{U}_{13}$ & $\mathrm{U}_{12}$ \\
\hline $\mathrm{N}(1)$ & $64(1)$ & $48(1)$ & $37(1)$ & $6(1)$ & $1(1)$ & $-1(1)$ \\
\hline $\mathrm{N}(2)$ & $62(1)$ & $39(1)$ & $56(1)$ & $-3(1)$ & $2(1)$ & $-3(1)$ \\
\hline $\mathrm{N}(3)$ & $48(1)$ & $54(1)$ & $34(1)$ & $-9(1)$ & $-2(1)$ & $8(1)$ \\
\hline$O(1) 1$ & $146(2)$ & $48(1)$ & $61(1)$ & $16(1)$ & $-13(1)$ & $-25(1)$ \\
\hline$O(2)$ & $72(1)$ & $66(1)$ & $39(1)$ & $9(1)$ & $-11(1)$ & $4(1)$ \\
\hline$O(3)$ & $82(1)$ & $51(1)$ & $65(1)$ & $-18(1)$ & $-8(1)$ & $-11(1)$ \\
\hline$O(4) 1$ & $125(1)$ & $43(1)$ & $72(1)$ & $11(1)$ & $-1(1)$ & $-12(1)$ \\
\hline$O(5)$ & $86(1)$ & $72(1)$ & $34(1)$ & $2(1)$ & $-10(1)$ & $6(1)$ \\
\hline$O(6)$ & $71(1)$ & $60(1)$ & $49(1)$ & $-17(1)$ & $-7(1)$ & $-4(1)$ \\
\hline$C(1)$ & $39(1)$ & $40(1)$ & $32(1)$ & $2(1)$ & $3(1)$ & $0(1)$ \\
\hline$C(2)$ & $37(1)$ & $43(1)$ & $32(1)$ & $-4(1)$ & $2(1)$ & $-1(1)$ \\
\hline$C(3)$ & $40(1)$ & $35(1)$ & $39(1)$ & $-3(1)$ & $4(1)$ & $-2(1)$ \\
\hline$C(4)$ & $41(1)$ & $43(1)$ & $33(1)$ & $3(1)$ & $4(1)$ & $4(1)$ \\
\hline$C(5)$ & $34(1)$ & $44(1)$ & $30(1)$ & $-4(1)$ & $4(1)$ & $1(1)$ \\
\hline$C(6)$ & $39(1)$ & $39(1)$ & $36(1)$ & $-4(1)$ & $5(1)$ & $-2(1)$ \\
\hline$C(7)$ & $42(1)$ & $61(1)$ & $37(1)$ & $1(1)$ & $4(1)$ & $1(1)$ \\
\hline$C(8)$ & $40(1)$ & $63(1)$ & $35(1)$ & $-10(1)$ & $3(1)$ & $-2(1)$ \\
\hline$C(9)$ & $44(1)$ & $57(1)$ & $43(1)$ & $-5(1)$ & $9(1)$ & $0(1)$ \\
\hline$C(10)$ & ) $43(1)$ & $64(1)$ & $33(1)$ & $-1(1)$ & $3(1)$ & $1(1)$ \\
\hline$C(11)$ & ) $43(1)$ & $62(1)$ & $39(1)$ & $-13(1)$ & $2(1)$ & $-3(1)$ \\
\hline$C(12)$ & ) 45 (1) & $53(1)$ & $48(1)$ & $-6(1)$ & $5(1)$ & $-3(1)$ \\
\hline$C(13)$ & $72(1)$ & $82(2)$ & $47(1)$ & $12(1)$ & $-1(1)$ & $4(1)$ \\
\hline$C(14)$ & ) $91(2)$ & $58(1)$ & $63(1)$ & $-2(1)$ & $8(1)$ & $-9(1)$ \\
\hline
\end{tabular}


Table 5. Hydrogen coordinates ( x 10 $0^{4}$ ) and isotropic displacement parameters $\left(\AA^{2} \times 10^{3}\right)$ for 4.

\begin{tabular}{lrrrr}
\hline & x & y & z & U(eq) \\
& & & & \\
\hline H (2) & 1702 & 4011 & 5612 & 45 \\
H (4) & -224 & 4353 & 390 & 47 \\
H (6) & -567 & 3004 & 2194 & 46 \\
H (8) & 5916 & 6599 & 9960 & 55 \\
H (10) & 3972 & 6571 & 4631 & 56 \\
H (11) & 3526 & 5767 & 4846 & 57 \\
H (12) & 4272 & 5371 & 7588 & 58 \\
H (13A) & 6156 & 5922 & 11786 & 100 \\
H (13B) & 6623 & 5458 & 10662 & 100 \\
H (13C) & 4565 & 5543 & 11296 & 100 \\
H (14A) & 4792 & 7305 & 5936 & 106 \\
H (14B) & 6489 & 7280 & 7454 & 106 \\
H (14C) & 4417 & 7347 & 8080 & 106 \\
& & & & \\
\hline
\end{tabular}


Table 1. Crystal data and structure refinement for 5 .

Identification code

Empirical formula

Formula weight

Density (calculated)

$\mathrm{F}(000)$

Temperature

Crystal size

Crystal color

Crystal description

Wavelength

Crystal system

Space group

Unit cell dimensions

Volume

$\mathrm{Z}$

Cell measurement reflections used

Cell measurement theta $\min / \max$

Diffractometer control software

Diffractometer measurement device

Diffractometer measurement method

Theta range for data collection

Completeness to theta $=28.29^{\circ}$

Index ranges

Computing data reduction

Absorption coefficient

Computing absorption correction

Absorption correction details

Max. / min. transmission

$\mathrm{R}$ (merg) before/after correction

Computing structure solution
5

$\mathrm{C}_{6} \mathrm{H}_{3} \mathrm{~N}_{3} \mathrm{O}_{6} * \mathrm{C}_{9} \mathrm{H}_{12}$

$333.30 \mathrm{Da}$

$1.392 \mathrm{~g} \mathrm{~cm}^{-3}$

696

183(2) K

$0.55 \times 0.35 \times 0.10 \mathrm{~mm}$

yellow

plate

$0.71073 \AA$

monoclinic

$P 2{ }_{1} / c$

$a=7.420(2) \AA$

$\alpha=90^{\circ}$

$b=7.555(2) \AA$

$\beta=93.013(7)^{\circ}$

$c=28.417(7) \AA$

$\gamma=90^{\circ}$

4

969

$2.718^{\circ}$ to $20.073^{\circ}$

Bruker AXS SMART Vers. 5.054 1997/98

Siemens SMART CCD area detector system

Full sphere data collection

in omega at $0.3^{\circ}$ scan width

two runs with 720 frames, phi $=0^{\circ}, 270^{\circ}$

and two runs with 436 frames, phi $=88^{\circ}, 180^{\circ}$

$2.79^{\circ}$ to $28.29^{\circ}$

$81.0 \%$

$-9<=\mathrm{h}<=9,-10<=\mathrm{k}<=9,-32<=\mathrm{l}<=37$

Bruker AXS SAINT program Vers. 6.02A

$0.110 \mathrm{~mm}^{-1}$

Bruker AXS SADABS program multiscan V2.03

R.H. Blessing, Acta Cryst. (1995) A51 33-38

$0.990 / 0.930$

$0.1476 / 0.0741$

Bruker AXS SHELXTL Vers. 6.12 DOS/WIN95/NT/2000/ME 
Computing structure refinement

Refinement method

Reflections collected

Independent reflections

Data / restraints / parameters

Goodness-of-fit on $\mathrm{F}^{2}$

Weighting details

Final $\mathrm{R}$ indices $[\mathrm{I}>2 \operatorname{sigma}(\mathrm{I})]$

$\mathrm{R}$ indices (all data)

Largest diff. peak and hole

Treatment of hydrogen atoms
Bruker AXS SHELXTL Vers. 6.12 DOS/WIN95/NT/2000/ME

Full-matrix least-squares on $\mathrm{F}^{2}$

6270

$3185[R(\mathrm{int})=0.0441]$

1587 / $0 / 217$

0.994

$\mathrm{w}=1 /\left[\sigma^{2}\left(\mathrm{Fo}^{2}\right)+(0.1092 * \mathrm{P})^{2}\right]$

where $\mathrm{P}=\left(\mathrm{Fo}^{2}+2 \mathrm{Fc}^{2}\right) / 3$

$R_{1}=0.0722, w R_{2}=0.1682$

$R_{1}=0.1391, w R_{2}=0.2126$

0.391 and $-0.189 \mathrm{e}^{-3}$

Riding model on idealized geometries

with the 1.2 fold (1.5 fold for methyl

groups) isotropic displacement

parameters of the equivalent Uij of the

corresponding carbon atom 
Table 2. Atomic coordinates ( $\left.\times 10^{4}\right)$ and equivalent isotropic displacement parameters $\left(\AA^{2} \times 10^{3}\right)$ for 5 . $U(e q)$ is defined as one third of the trace of the orthogonalized $U_{i j}$ tensor.

\begin{tabular}{|c|c|c|c|c|}
\hline & $\mathrm{x}$ & $\mathrm{y}$ & $\mathrm{z}$ & $\mathrm{U}(\mathrm{eq})$ \\
\hline $\mathrm{N}(1)$ & $5040(4)$ & $8932(3)$ & $2954(1)$ & $59(1)$ \\
\hline $\mathrm{N}(2)$ & $6466(4)$ & $7394(4)$ & $4599(1)$ & $59(1)$ \\
\hline $\mathrm{N}(3)$ & $3565(4)$ & $3009(3)$ & $3532(1)$ & $55(1)$ \\
\hline$O(1)$ & $4036(4)$ & $8650(3)$ & $2603(1)$ & $82(1)$ \\
\hline$O(2)$ & $6050(3)$ & $10196(3)$ & 3005 (1) & $78(1)$ \\
\hline$O(3)$ & $6931(4)$ & $8932(3)$ & $4662(1)$ & $86(1)$ \\
\hline$O(4)$ & $6583(3)$ & $6242(4)$ & $4902(1)$ & $79(1)$ \\
\hline$O(5)$ & $3723(3)$ & $1950(3)$ & $3860(1)$ & $74(1)$ \\
\hline$O(6)$ & $2845(3)$ & $2695(3)$ & $3148(1)$ & $73(1)$ \\
\hline$C(1)$ & $5026(4)$ & $7625(3)$ & $3337(1)$ & $43(1)$ \\
\hline$C(2)$ & $5761(4)$ & $8110(3)$ & $3772(1)$ & $44(1)$ \\
\hline$C(3)$ & $5730(4)$ & $6882(3)$ & $4131(1)$ & $43(1)$ \\
\hline$C(4)$ & $5005(3)$ & $5212(3)$ & $4063(1)$ & $43(1)$ \\
\hline$C(5)$ & $4309(3)$ & $4804(3)$ & $3619(1)$ & $41(1)$ \\
\hline$C(6)$ & $4276(4)$ & $5978(3)$ & $3249(1)$ & $43(1)$ \\
\hline$C(7)$ & $-173(4)$ & $6765(4)$ & $3333(1)$ & $56(1)$ \\
\hline$C(8)$ & $555(4)$ & $7780(4)$ & $3704(1)$ & $53(1)$ \\
\hline$C(9)$ & $764(4)$ & $7108(4)$ & $4158(1)$ & $50(1)$ \\
\hline$C(10)$ & $220(4)$ & $5367(4)$ & $4231(1)$ & $52(1)$ \\
\hline$C(11)$ & $-515(4)$ & $4341(4)$ & $3870(1)$ & $55(1)$ \\
\hline$C(12)$ & $-707(4)$ & $5046(4)$ & $3428(1)$ & $56(1)$ \\
\hline$C(13)$ & $-369(6)$ & $7469(7)$ & $2840(1)$ & $99(2)$ \\
\hline$C(14)$ & $1552(5)$ & $8201(5)$ & $4561(1)$ & $79(1)$ \\
\hline$C(15)$ & $-1094(5)$ & $2447(4)$ & $3971(2)$ & $93(1)$ \\
\hline
\end{tabular}


Table 3. Bond lengths $[\AA]$ and angles $\left[^{\circ}\right]$ for 5.

\begin{tabular}{|c|c|c|c|}
\hline $\mathrm{N}(1)-\mathrm{O}(2)$ & $1.218(3)$ & $O(3)-N(2)-C(3)$ & $118.2(3)$ \\
\hline $\mathrm{N}(1)-\mathrm{O}(1)$ & $1.233(4)$ & $\mathrm{O}(4)-\mathrm{N}(2)-\mathrm{C}(3)$ & $117.5(3)$ \\
\hline $\mathrm{N}(1)-\mathrm{C}(1)$ & $1.469(4)$ & $\mathrm{O}(6)-\mathrm{N}(3)-\mathrm{O}(5)$ & $125.1(3)$ \\
\hline$N(2)-O(3)$ & $1.222(3)$ & $O(6)-N(3)-C(5)$ & $118.2(3)$ \\
\hline $\mathrm{N}(2)-\mathrm{O}(4)$ & $1.225(3)$ & $O(5)-N(3)-C(5)$ & $116.7(3)$ \\
\hline $\mathrm{N}(2)-\mathrm{C}(3)$ & $1.463(4)$ & $C(2)-C(1)-C(6)$ & $122.7(3)$ \\
\hline$N(3)-O(6)$ & $1.213(3)$ & $\mathrm{C}(2)-\mathrm{C}(1)-\mathrm{N}(1)$ & $118.0(3)$ \\
\hline$N(3)-O(5)$ & $1.231(3)$ & $\mathrm{C}(6)-\mathrm{C}(1)-\mathrm{N}(1)$ & $119.3(3)$ \\
\hline$N(3)-C(5)$ & $1.480(4)$ & $C(1)-C(2)-C(3)$ & $117.7(3)$ \\
\hline$C(1)-C(2)$ & $1.373(4)$ & $C(2)-C(3)-C(4)$ & $122.3(3)$ \\
\hline$C(1)-C(6)$ & $1.381(4)$ & $C(2)-C(3)-N(2)$ & $118.3(3)$ \\
\hline$C(2)-C(3)$ & $1.380(4)$ & $C(4)-C(3)-N(2)$ & $119.4(3)$ \\
\hline$C(3)-C(4)$ & $1.381(4)$ & $C(5)-C(4)-C(3)$ & $117.2(3)$ \\
\hline$C(4)-C(5)$ & $1.374(4)$ & $C(6)-C(5)-C(4)$ & $123.3(3)$ \\
\hline$C(5)-C(6)$ & $1.374(4)$ & $C(6)-C(5)-N(3)$ & $118.1(3)$ \\
\hline$C(7)-C(12)$ & $1.388(4)$ & $C(4)-C(5)-N(3)$ & $118.6(3)$ \\
\hline$C(7)-C(8)$ & $1.390(4)$ & $C(5)-C(6)-C(1)$ & $116.9(3)$ \\
\hline$C(7)-C(13)$ & $1.499(5)$ & $C(12)-C(7)-C(8)$ & $118.2(3)$ \\
\hline$C(8)-C(9)$ & $1.387(4)$ & $C(12)-C(7)-C(13)$ & $119.9(3)$ \\
\hline$C(9)-C(10)$ & $1.395(4)$ & $C(8)-C(7)-C(13)$ & $121.9(3)$ \\
\hline$C(9)-C(14)$ & $1.505(4)$ & $C(9)-C(8)-C(7)$ & $121.6(3)$ \\
\hline$C(10)-C(11)$ & $1.374(4)$ & $C(8)-C(9)-C(10)$ & $117.6(3)$ \\
\hline$C(11)-C(12)$ & $1.367(4)$ & $C(8)-C(9)-C(14)$ & $121.8(3)$ \\
\hline \multirow[t]{2}{*}{$C(11)-C(15)$} & $1.526(4)$ & $C(10)-C(9)-C(14)$ & $120.5(3)$ \\
\hline & & $C(11)-C(10)-C(9)$ & $121.9(3)$ \\
\hline $\mathrm{O}(2)-\mathrm{N}(1)-\mathrm{O}(1)$ & $124.9(3)$ & $C(12)-C(11)-C(10)$ & $119.0(3)$ \\
\hline $\mathrm{O}(2)-\mathrm{N}(1)-\mathrm{C}(1)$ & $117.9(3)$ & $C(12)-C(11)-C(15)$ & $121.4(3)$ \\
\hline $\mathrm{O}(1)-\mathrm{N}(1)-\mathrm{C}(1)$ & $117.2(3)$ & $C(10)-C(11)-C(15)$ & $119.6(3)$ \\
\hline$O(3)-N(2)-O(4)$ & $124.3(3)$ & $C(11)-C(12)-C(7)$ & $121.7(3)$ \\
\hline
\end{tabular}

Symmetry transformations used to generate equivalent atoms: 
Table 4. Anisotropic displacement parameters $\left(\AA^{2} \times 10^{3}\right)$ for 5 . The anisotropic displacement factor exponent takes the form: $-2 \pi^{2}\left[h^{2} a^{* 2} U_{11}+\ldots+2 h \mathrm{k}^{*} b^{*} U_{12}\right]$

\begin{tabular}{|c|c|c|c|c|c|c|}
\hline & $\mathrm{U}_{11}$ & $\mathrm{U}_{22}$ & $\mathrm{U}_{33}$ & $\mathrm{U}_{23}$ & $\mathrm{U}_{13}$ & $\mathrm{U}_{12}$ \\
\hline $\mathrm{N}(1)$ & $65(2)$ & $58(2)$ & $56(2)$ & $11(1)$ & $9(2)$ & $12(1)$ \\
\hline $\mathrm{N}(2)$ & $49(2)$ & $76(2)$ & $50(2)$ & $-8(2)$ & $0(1)$ & $-6(1)$ \\
\hline $\mathrm{N}(3)$ & $45(2)$ & $43(1)$ & $78(2)$ & $-11(1)$ & $6(2)$ & $2(1)$ \\
\hline$O(1) 1$ & $103(2)$ & $85(2)$ & $59(2)$ & $18(1)$ & $-1(2)$ & $6(1)$ \\
\hline$O(2)$ & $90(2)$ & $56(1)$ & $90(2)$ & $20(1)$ & $16(2)$ & $-8(1)$ \\
\hline$O(3)$ & $99(2)$ & $79(2)$ & $79(2)$ & $-30(1)$ & $-12(2)$ & $-18(1)$ \\
\hline$O(4)$ & $78(2)$ & $107(2)$ & $50(1)$ & $5(1)$ & $-10(1)$ & $-13(1)$ \\
\hline$O(5)$ & $83(2)$ & $42(1)$ & $97(2)$ & $6(1)$ & $11(2)$ & $-6(1)$ \\
\hline$O(6)$ & $73(2)$ & $64(2)$ & $81(2)$ & $-30(1)$ & $-11(2)$ & $-8(1)$ \\
\hline$C(1)$ & $42(2)$ & $43(2)$ & $46(2)$ & $1(1)$ & $7(2)$ & $5(1)$ \\
\hline$C(2)$ & $39(2)$ & $41(1)$ & $53(2)$ & $-3(1)$ & $8(2)$ & $0(1)$ \\
\hline$C(3)$ & $38(2)$ & $49(2)$ & $43(2)$ & $-6(1)$ & $2(1)$ & $0(1)$ \\
\hline$C(4)$ & $40(2)$ & $42(2)$ & $47(2)$ & $2(1)$ & $5(2)$ & $1(1)$ \\
\hline$C(5)$ & $36(2)$ & $37(1)$ & $52(2)$ & $-7(1)$ & $3(1)$ & $3(1)$ \\
\hline$C(6)$ & $38(2)$ & $50(2)$ & $42(2)$ & $-8(1)$ & $1(1)$ & $8(1)$ \\
\hline$C(7)$ & $46(2)$ & $72(2)$ & $52(2)$ & $1(2)$ & $5(2)$ & $19(2)$ \\
\hline$C(8)$ & $43(2)$ & $43(2)$ & $74(2)$ & $2(2)$ & $12(2)$ & $9(1)$ \\
\hline$C(9)$ & $37(2)$ & $51(2)$ & $61(2)$ & $-14(2)$ & $0(2)$ & $6(1)$ \\
\hline$C(10)$ & $41(2)$ & $56(2)$ & $58(2)$ & $4(2)$ & $4(2)$ & $4(1)$ \\
\hline$C(11)$ & $43(2)$ & $47(2)$ & $75(2)$ & $-10(2)$ & $7(2)$ & $6(1)$ \\
\hline$C(12)$ & $40(2)$ & $61(2)$ & $65(2)$ & $-25(2)$ & $-3(2)$ & $3(1)$ \\
\hline$C(13)$ & $83(3)$ & $144(4)$ & $70(3)$ & $29(3)$ & $8(2)$ & $37(3)$ \\
\hline$C(14)$ & $61(2)$ & $89(3)$ & $86(3)$ & $-42(2)$ & $2(2)$ & $-1(2)$ \\
\hline$C(15)$ & $77(3)$ & $48(2)$ & $157(4)$ & $-12(2)$ & $23(3)$ & $-4(2)$ \\
\hline
\end{tabular}


Table 5. Hydrogen coordinates ( x 10 $0^{4}$ ) and isotropic displacement parameters $\left(\AA^{2} \times 10^{3}\right)$ for 5.

\begin{tabular}{lrrrr}
\hline & x & y & z & U(eq) \\
& & & & \\
& & & & 53 \\
H (2) & 6274 & 9249 & 3823 & 52 \\
H (4) & 4986 & 4382 & 4314 & 52 \\
H (6) & 3762 & 5670 & 2948 & 64 \\
H (8) & 917 & 8961 & 3646 & 62 \\
H (10) & 365 & 4876 & 4538 & 67 \\
H (12) & -1220 & 4342 & 3178 & 149 \\
H (13A) & 61 & 8694 & 2835 & 149 \\
H (13B) & -1642 & 7431 & 2729 & 118 \\
H (13C) & 345 & 6746 & 2632 & 118 \\
H (14A) & 1856 & 9382 & 4447 & 118 \\
H (14B) & 2645 & 7625 & 4695 & 140 \\
H (14C) & 668 & 8305 & 4803 & 140 \\
H (15A) & -1593 & 1903 & 3680 & 140 \\
H (15B) & -2013 & 2455 & 4206 & \\
H (15C) & -45 & 1766 & 4091 & \\
\hline
\end{tabular}




\section{DSC}

The melting points of the crystals of solvates 1-5 were recorded on NETZSCH, Phoenix DSC 2004. The thermograms are shown below. There were no additional peaks in the thermograms indicating phase transition.

Diffrential Scanning thermograms of 1-5

1

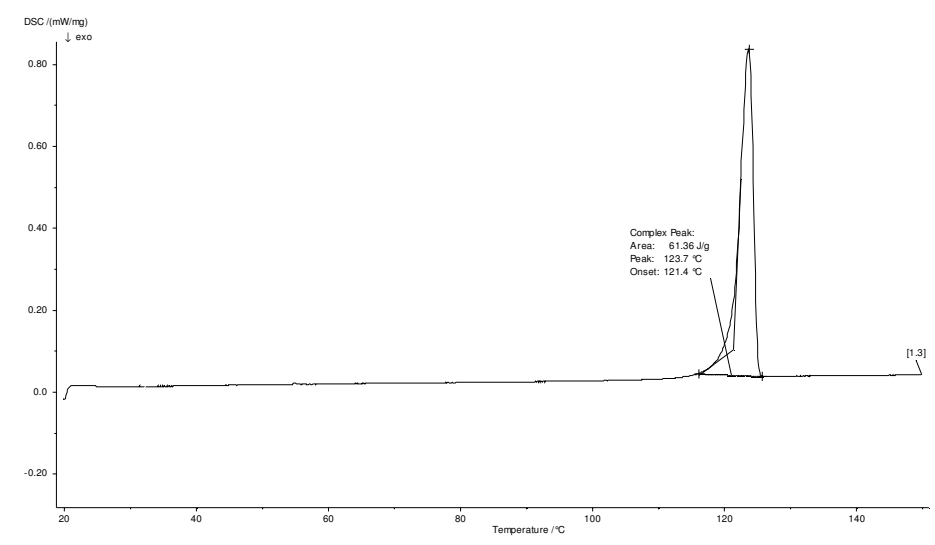

2

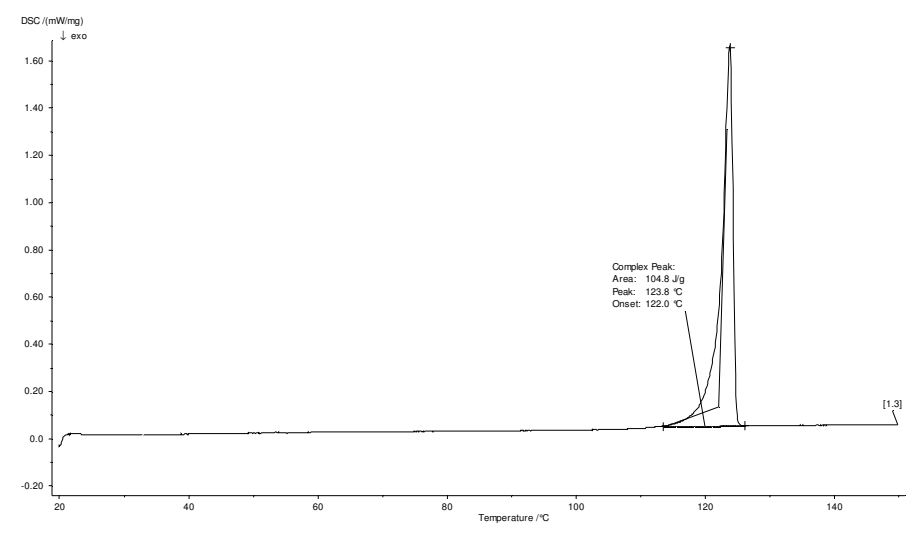

3

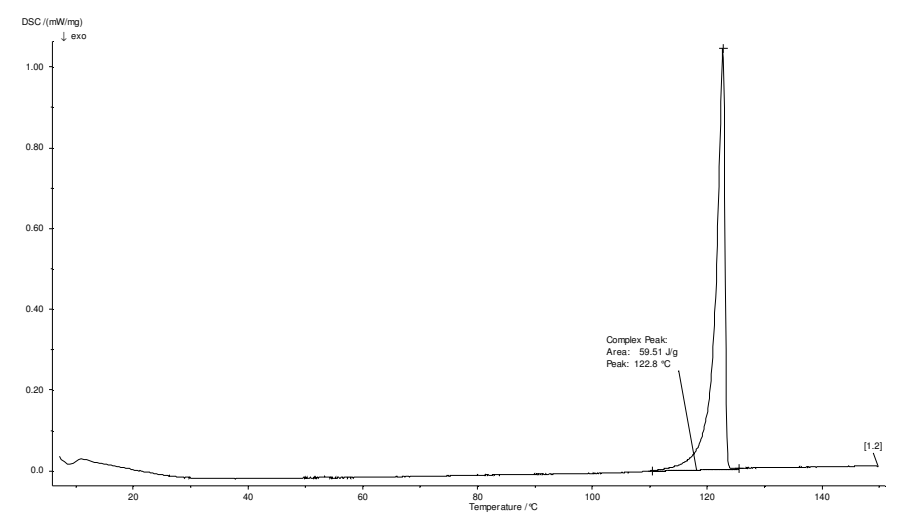



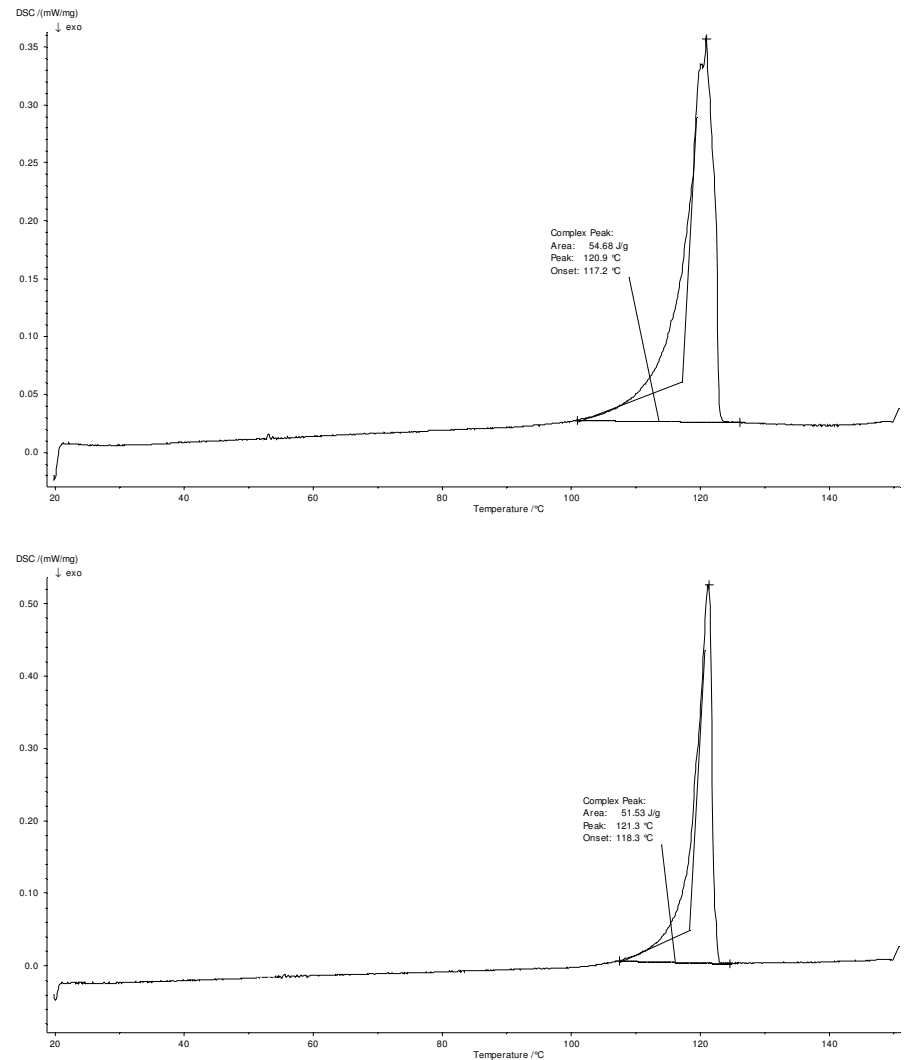